THE AstronOMiCAL JouRNAL, 120:2904-2919, 2000 December

(C) 2000. The American Astronomical Society. All rights reserved. Printed in U.S.A.

\title{
HIGH SPATIAL RESOLUTION IMAGING OF NGC 1068 IN THE MID-INFRARED
}

\author{
J. J. Bock, ${ }^{1,2}$ G. Neugebauer, ${ }^{3}$ K. Matthews, ${ }^{3}$ B. T. Soifer, ${ }^{3,4}$ E. E. Becklin, ${ }^{5}$ M. Ressler, ${ }^{2}$ \\ K. MARSh, ${ }^{6}$ M. W. Werner, ${ }^{2}$ E. EGAMI, ${ }^{3}$ AND R. BlandFORD ${ }^{1}$ \\ Received 2000 July 21 ; accepted 2000 September 6
}

\begin{abstract}
Mid-infrared observations of the central source of NGC 1068 have been obtained with a spatial resolution in the deconvolved image of $0.1(\sim 7 \mathrm{pc})$. The central source is extended by $\sim 1^{\prime \prime}$ in the northsouth direction but appears unresolved in the east-west direction over most of its length. About $2 / 3$ of its flux can be ascribed to a core structure that is itself elongated north-south and does not show a distinct unresolved compact source. The source is strongly asymmetric, extending significantly farther to the north than to the south. The morphology of the mid-infrared emission appears similar to that of the radio jet and has features which correlate with the images in [O III]. Its 12.5-24.5 $\mu \mathrm{m}$ color temperature ranges from 215 to $260 \mathrm{~K}$ and does not decrease smoothly with distance from the core. Silicate absorption is strongest in the core and to the south and is small in the north. The core, apparently containing $2 / 3$ of the bolometric luminosity of the inner 4 " diameter area, may be explained by a thick, dusty torus near the central active galactic nucleus (AGN) viewed at an angle of $\sim 65^{\circ}$ to its plane. There are, however, detailed difficulties with existing models, especially the narrow east-west width of the thin extended mid-infrared "tongue" to the north of the core. We interpret the tongue as reprocessed visual and ultraviolet radiation that is strongly beamed and that originates in the AGN.
\end{abstract}

Key words: galaxies: individual (NGC 1068) - infrared radiation

\section{INTRODUCTION}

NGC 1068 is a nearby heavily obscured Seyfert 2 galaxy with a bright active galactic nucleus (AGN) that has been studied in nearly every available wavelength band and at many spatial resolutions. Its classification as a Seyfert 2 nucleus rests on the basis of narrow emission lines. Antonucci \& Miller (1985) have, however, discovered broad, polarized emission lines indicative of a broad-line region, which suggests the presence of a Seyfert 1 nucleus hidden by a thick circumnuclear torus or a heavily warped disk, a possibility we shall henceforth include in the word "torus." Barthel (1989) suggested that NGC 1068 might be a prototype for many classes of AGNs. Thus NGC 1068 has provided the framework for a unification scheme based on a dense disk of molecular gas and dust surrounding a broadline region and Seyfert 1 nucleus; see, e.g., Krolik (1999) and references therein. In this model, Seyfert 1 and 2 galaxies are substantially similar objects viewed at different orientations. An alternative to the torus geometry that has been suggested by Cameron et al. (1993) is that the molecular material in the nuclear vicinity is distributed in such a way that the bulk of the gas and dust lies $\sim 100 \mathrm{pc}$ or so from the nucleus. We take the distance to NGC 1068 as $14.4 \mathrm{Mpc}$

${ }^{1}$ Division of Physics, Math and Astronomy, California Institute of Technology, Pasadena, CA 91125; jjb@astro.caltech.edu, rdb@tapir.caltech.edu.

2 Jet Propulsion Lab, 169-327, 4800 Oak Grove Drive, Pasadena, CA 91109; ressler@cougar.jpl.nasa.gov,mww@ipac.caltech.edu.

${ }^{3}$ Palomar Observatory, California Institute of Technology, 105-24, Pasadena,CA 91125; gxn@caltech.edu,kym@caltech.edu,

bts@mop.caltech.edu, egami@mop.caltech.edu.

${ }^{4}$ SIRTF Science Center, California Institute of Technology 314-6, Pasadena, CA 91125.

${ }_{5}^{5}$ Department of Physics and Astronomy, UCLA, Los Angeles, CA 90095-1562; becklin@astro.ucla.edu.

${ }^{6}$ IPAC, Jet Propulsion Lab/ California Institute of Technology, 100-22, Pasadena, CA 91125; kam@ipac.caltech.edu.
(Tully 1988) so that $1^{\prime \prime}=72 \mathrm{pc}$; we assume $H_{0}$ to be $75 \mathrm{~km}$ $\mathrm{s}^{-1} \mathrm{Mpc}^{-1}$ throughout this paper.

Since Antonucci \& Miller (1985) suggested that NGC 1068 contains an obscuring torus, there have been numerous theoretical studies of thick tori in AGNs. Three that have focused on NGC 1068, mainly constrained by the energetics, are those of Efstathiou, Hugh, \& Young (1995), Pier \& Krolik (1993), and Granato, Danese, \& Franceschini (1997); these give numerous references to other theoretical models and issues.

Recently, observations of the nucleus of NGC 1068 at a range of wavelengths with high spatial resolution have been published. Differences exist between different images, but most find strong nuclear emission in a roughly north-south direction; the putative torus is presumed to lie in roughly an east-west plane. Hubble Space Telescope (HST) observations of the inner $3^{\prime \prime}$ of NGC 1068 in an [O III] filter by Evans et al. (1991) and Macchetto et al. (1994) resolve the narrow-line region into a conical shape oriented northeast by southwest. Spectral observations by Crenshaw \& Kraemer (2000a, 2000b) and Kraemer \& Crenshaw (2000) of the [O III]-emitting gas have further delineated the cone. Speckle observations at the $2.2 \mu \mathrm{m}$ diffraction limit of the Keck 10 meter Telescope show that about half of the nuclear flux at $2.2 \mu \mathrm{m}$ within a diameter of $\sim 1^{\prime \prime}$ comes from an extended region, essentially north-south but orientated slightly west of north, 0 .'3 (22 pc) in length on either side of a point source which is less than 0.03 (2 pc) in size (Weinberger, Neugebauer, \& Matthews 1999). Rouan et al. (1998) have used adaptive optics to obtain images at 1.2, 1.6, and $2.2 \mu \mathrm{m}$ with higher dynamic range but poorer spatial resolution than Weinberger et al. and see evidence of elongated structure at a position angle of P.A. $\approx 102^{\circ}$, which might be tracing the putative torus. Marco \& Alloin (2000), observing at 3.5 and $4.8 \mu \mathrm{m}$, see an unresolved core with a full width at half-maximum (FWHM) less than $0.2(16 \mathrm{pc})$, a north-south elongation about $1^{\prime \prime}(70 \mathrm{pc})$ in length, plus $\mathrm{a}$ "disklike" structure with a diameter $\sim 1$ ".0 $(70 \mathrm{pc})$ at a 
TABLE 1

LOG OF OBSERVATIONS

\begin{tabular}{|c|c|c|c|c|c|c|c|}
\hline Object Number & Image & $7.9 \mu \mathrm{m}$ & $10.3 \mu \mathrm{m}$ & $12.5 \mu \mathrm{m}$ & $24.5 \mu \mathrm{m}$ & UT & Air Mass \\
\hline \multicolumn{8}{|c|}{1998 Oct 1} \\
\hline NGC $1068 \ldots . .$. & 1 & & & $\sqrt{ }$ & & 10:09 & 1.27 \\
\hline HR $0911 \ldots \ldots .$. & 1 & $\sqrt{ }$ & & $\sqrt{ }$ & $\sqrt{ }$ & $10: 21$ & 1.26 \\
\hline HR $0911 \ldots \ldots .$. & 2 & $\sqrt{ }$ & & $\sqrt{ }$ & $\sqrt{ }$ & $10: 54$ & 1.16 \\
\hline NGC $1068 \ldots \ldots$ & 2 & $\sqrt{ }$ & & $\sqrt{ }$ & $\sqrt{ }$ & $11: 17$ & 1.11 \\
\hline HR $0911 \ldots \ldots .$. & 3 & $\sqrt{ }$ & & $\sqrt{ }$ & $\sqrt{ }$ & $11: 43$ & 1.08 \\
\hline NGC $1068 \ldots . .$. & 3 & $\sqrt{ }$ & & $\sqrt{ }$ & $\sqrt{ }$ & $12: 14$ & 1.06 \\
\hline HR $1457 \ldots \ldots \ldots$ & 4 & $\sqrt{ }$ & & $\sqrt{ }$ & $\sqrt{ }$ & $12: 46$ & 1.08 \\
\hline NGC $1068 \ldots . .$. & 4 & $\sqrt{ }$ & & $\sqrt{ }$ & $\sqrt{ }$ & $13: 15$ & 1.09 \\
\hline HR $1457 \ldots \ldots \ldots$ & 5 & $\sqrt{ }$ & & $\sqrt{ }$ & $\sqrt{ }$ & $13: 33$ & 1.01 \\
\hline \multicolumn{8}{|c|}{1998 Oct 3} \\
\hline HR $0911 \ldots \ldots .$. & 6 & & $\sqrt{ }$ & $\sqrt{ }$ & & $12: 32$ & 1.03 \\
\hline NGC $1068 \ldots . .$. & 6 & & $\sqrt{ }$ & $\sqrt{ }$ & & $12: 50$ & 1.08 \\
\hline HR $0911 \ldots \ldots .$. & 7 & & & $\sqrt{ }$ & & $13: 18$ & 1.05 \\
\hline NGC $1068 \ldots . .$. & 7 & & $\sqrt{ }$ & $\sqrt{ }$ & & $14: 13$ & 1.22 \\
\hline
\end{tabular}

P.A. $\sim 100^{\circ}$, which they interpret as the dusty torus invoked in unification schemes.

Gallimore et al. (1996b) and Gallimore, Baum, \& O'Dea (1996a) have summarized the subarcsecond radio structure of NGC 1068 as consisting of three components in a northeast by southwest line. A $10^{\prime \prime}$ (kiloparsec) scale radio jet joins the southern and central radio components and traces emission to subarcsecond (tens of parsecs) scales. The southernmost radio peak consists of two clumps, the brighter of which has a relatively flat radio spectrum and is associated with $\mathrm{H}_{2} \mathrm{O}$ and $\mathrm{OH}$ masers characterizing warm and dense molecular gas and showing Keplerian motion. Gallimore et al. (1996a) consider this region (called S1) as most likely being at the location of the central engine of NGC 1068 because of these features. Gallimore, Baum, \& O'Dea (1997) have imaged $\mathrm{S} 1$ at $8.4 \mathrm{GHz}$ and give the first direct image of a parsec-scale ionized gas disk surrounding the AGN in NGC 1068 when they trace a set of compact sources of overall extent $0^{\prime \prime} .15$ at a position angle of $\sim 110^{\circ}$. S1 appears to also coincide with the apex of the [O III] cone within the astrometric uncertainties between the visual and radio images (0".2; Evans et al. 1991). At millimeter wavelengths, Schinnerer et al. (2000) see two emission knots in the ${ }^{12} \mathrm{CO}(2-1)$ line about $1^{\prime \prime}$ east and west of the nucleus surrounded by a ring of emission with radius $\sim 1.5^{\prime \prime}(100$ pc).

The bolometric luminosity of NGC 1068 is determined mainly in the mid-infrared where it is extremely bright; its flux distribution peaks at $\sim 25 \mu \mathrm{m}$. If the infrared emission is isotropic, the bolometric luminosity of NGC 1068 is $\sim 2.5-3.0 \times 10^{11} L_{\odot}$, most of which is emitted between 10 and $100 \mu \mathrm{m}$. This includes, however, the emission from the " 3 kpc ring," a disk about 30 " in diameter, which was observed at 10,60, and $158 \mu \mathrm{m}$ by Telesco et al. (1984) and found to account for almost half the luminosity of NGC 1068. Telesco et al. attributed this emission to a molecular cloud with ongoing star formation. Subsequent radio measurements by Planesas, Scoville, \& Myers (1991) and Papadopoulos, Seaquist, \& Scoville (1997) further delineated the properties of the molecular cloud. Finally, Laurent et al. (2000) obtained CVF spectra between 5 and $16 \mu \mathrm{m}$, which allowed them to separate clearly the mid-infrared emission from the nucleus and from the outer ring. Based on the observations detailed below we will take the bolometric luminosity of the central area 4" in diameter of NGC 1068 to be $1.5 \times 10^{11} L_{\odot}$.

Hard X-ray observations of NGC 1068 provide another estimate of the intrinsic AGN luminosity. A strong Fe K line, first detected with Ginga by Koyama et al. (1989), indicates that the observed X-rays are scattered and reflected emission and that the direct line of sight to the AGN is completely blocked by a Compton-thick material, a result confirmed by observations with $A S C A$ (Ueno et al. 1994) and BeppoSAX (Matt et al. 1997). The intrinsic 2-10 keV luminosity of NGC 1068 is estimated to be on the order of $\sim 1.1 \times 10^{10} L_{\odot}$ (Iwasawa, Fabian, \& Matt 1997; rescaled to $H_{0}=75 \mathrm{~km} \mathrm{~s}^{-1} \mathrm{Mpc}^{-1}$ ). If $L_{\text {bol }} \sim 30 L_{2-10 \mathrm{kev}}$ (Matt et al. 2000), the intrinsic AGN bolometric luminosity is estimated to be $3 \times 10^{11} L_{\odot}$.

The reduced obscuration at mid-infrared wavelengths allows a detailed examination of the nuclear structure and means that mid-infrared observations are crucial in determining the morphology of the nucleus. The previous midinfrared observations of NGC 1068 with the highest spatial resolution are those of Bock et al. (1998) who made diffraction-limited observations (FWHM $\sim 0$ ".5) with the Hale 200 inch $(5 \mathrm{~m})$ Telescope at $8.8,10.3$, and $12.5 \mu \mathrm{m}$; see Bock et al. for references to previous mid-infrared work. Bock et al. report a central peak with structures extending about $1^{\prime \prime}$ north-south which is unresolved east-west and coincides with one wall of the ionization cone seen by Evans et al. (1991). In this paper we report similar observations but made at wavelengths from 7.9 to $24.5 \mu \mathrm{m}$ with the 10 meter Keck Telescope and accordingly having twice the raw spatial resolution as the Palomar observations. The present observations were limited to the inner $4^{\prime \prime}$ of the galaxy and pertain to this extreme nuclear region only.

\section{OBSERVATIONS}

Observations of NGC 1068 were conducted from the Keck 210 meter Telescope on the nights of 1998 October 1 and October3 with the mid-infrared camera MIRLIN (Ressler et al. 1994). MIRLIN is based on the HF-16 $128 \times 128 \mathrm{Si}$ :As BIB focal plane array with a plate scale at the Keck Telescope of 0.138 pixel $^{-1}$, resulting in Nyquistsampling of the diffraction-limited Airy function for $\lambda>12$ 


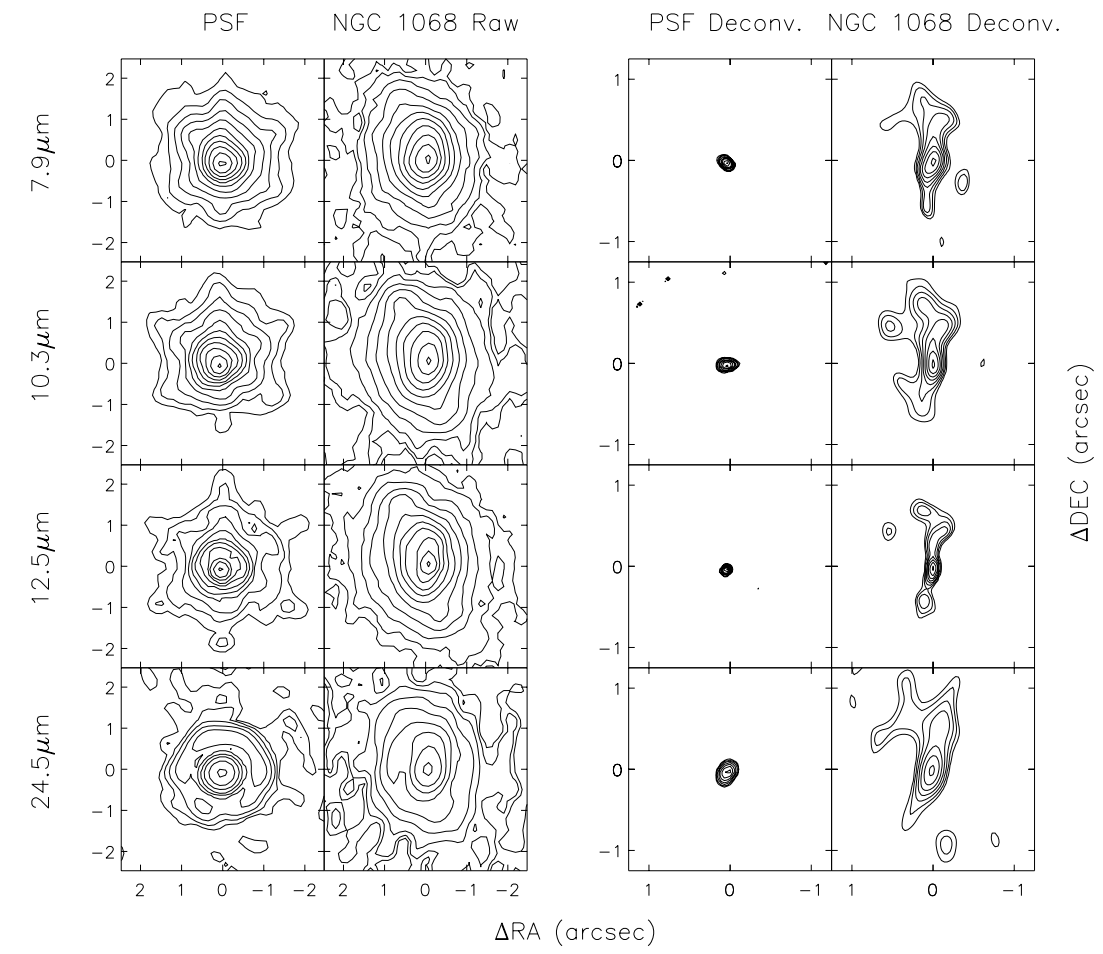

FIG. $1 a$

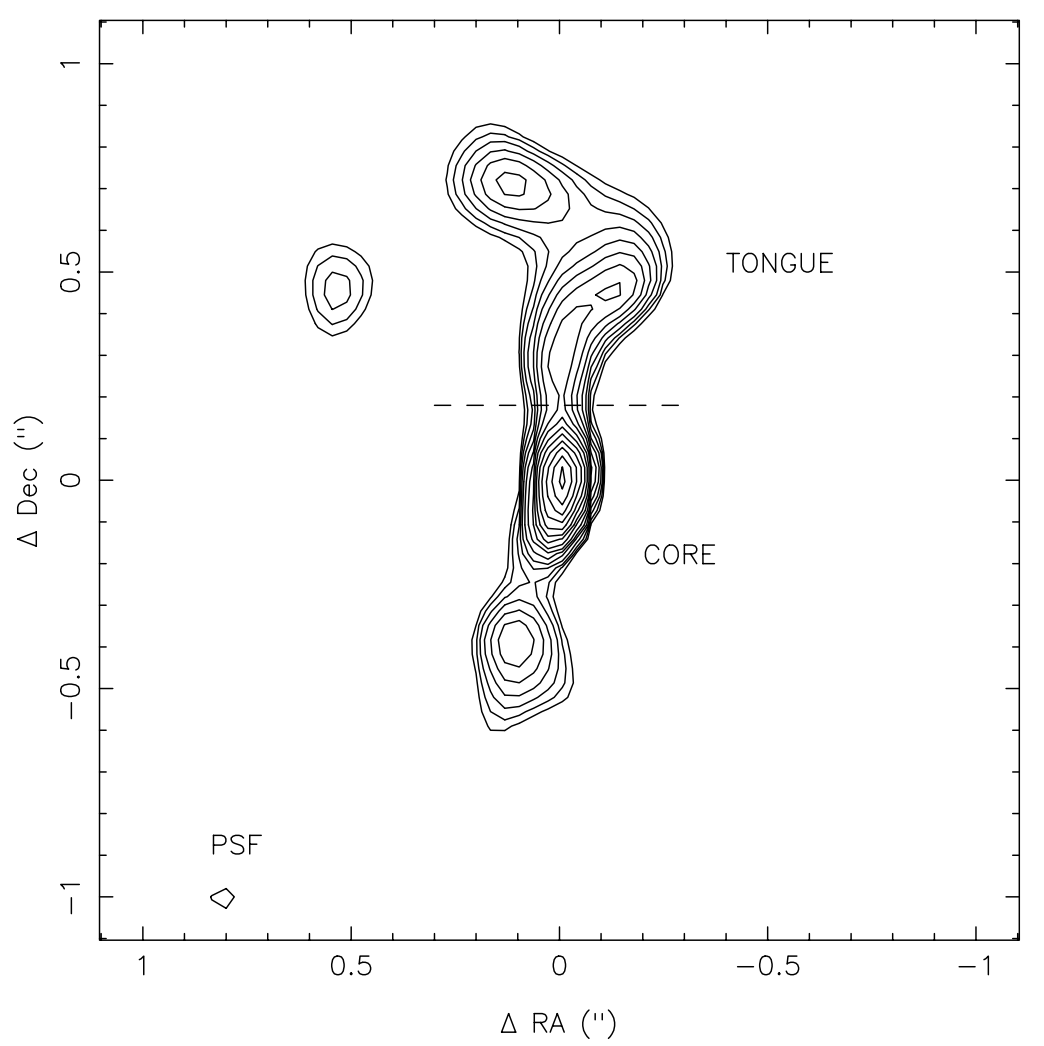

FIG. $1 b$

FIG. 1.-(a) Four images from the present data are given for wavelengths $7.9 \mu \mathrm{m}$ (top row), $10.3 \mu \mathrm{m}$ (second row), $12.5 \mu \mathrm{m}$ (third row), and $24.5 \mu \mathrm{m}$ (bottom row). The first two columns show the raw images of the appropriate PSF and of NGC 1068. The two right-hand columns show the deconvolved images of the appropriate PSF and of NGC 1068. Note that the size scales are different between the raw and deconvolved images. North is up and east is to the left in all the images. The maximum contour shown is of pixels with a flux density surface brightness of 0.9 times maximum flux density surface brightness per pixel. The minimum contour shown is down by a factor of 140 from the peak surface brightness in the 7.9,10.3, and $12.5 \mu \mathrm{m}$ images and by a factor of 70 in the 24.5 $\mu \mathrm{m}$ image. Contour levels are spaced by multiplicative factors of 2. (b) Deconvolved image at $12.5 \mu \mathrm{m}$, which has the highest spatial resolution and the highest signal-to-noise ratio, i.e., the highest dynamic range, is duplicated at a larger scale than in $(a)$. The contour spacing in the NGC 1068 image is $\sqrt{2}$; the other parameters of the contour levels are as in $(a)$. The contour for the PSF is drawn at half of the maximum flux pixel ${ }^{-1}$. The dashed horizontal line marks the arbitrary demarcation between the core - to the south - and the tongue - to the north. 
$\mu \mathrm{m}$. In order to characterize and monitor the point spread function and to obtain photometric calibration, observations of NGC 1068 were interleaved with those of a bright infrared stellar source, either BS $=$ HR $0911(\alpha$ Ceti) or BS $=$ HR 1457 ( $\alpha$ Tauri). The sequence of observations shown in Table 1 was chosen so that the infrared standards were observed at a similar elevation angle as NGC 1068. The targets were observed in four mid-infrared spectral bands, centered on $7.91 \mu \mathrm{m}(\Delta \lambda=0.76 \mu \mathrm{m}), 10.27 \mu \mathrm{m}$ $(\Delta \lambda=1.01 \mu \mathrm{m}), 12.49 \mu \mathrm{m}(\Delta \lambda=1.16 \mu \mathrm{m})$, and $24.48 \mu \mathrm{m}$ $(\Delta \lambda=0.76 \mu \mathrm{m})$.

In order to subtract thermal emission accurately from the atmosphere and telescope, each target was observed with a standard "chop-and-nod" strategy. A chopped image was created by co-adding exposures in the on and off beam of the chopping secondary mirror. The telescope was then nodded to remove any offset induced by the chopper, and the observation repeated to create the "chop-nodded" image. Typical integration times were $80-100 \mathrm{~ms}$ in each beam of the chopper, with a total of 4-5 s of integration on the sky spent on each of the four images. Table 1 is a log of the observations. Each observation listed in Table 1 consisted of a sequence of at least three chop-nodded images at each wavelength, dithered by $\sim 0$ ".7 between images in order to sample different pixels on the array. The telescope was guided during the observations on nearby stars using an automated optical guider, always selecting the guide star so as to avoid diffraction spikes from the target. The chopand-nod amplitudes were each 5" so as to keep the image of the target on the focal plane array for maximum observing efficiency. To avoid any smearing of the images during the exposures, the settling time during each chopper cycle was $15 \mathrm{~ms}$, and the settling time between nod positions was $15 \mathrm{~s}$. In order to confirm the PSF was not elongated due to chopping or nodding, the chopping was north-south and nodding east-west on 1998 October 1 , and the chopping was east-west and nodding north-south on 1998 October 3.

\section{DATA REDUCTION AND ANALYSIS}

\subsection{Data Reduction}

The raw data of NGC 1068 and the PSF calibration stars were combined by dividing each exposure by a flat-field frame and interpolating over bad pixels from a bad pixel mask. The four subimages in each chopped-nodded frame were combined, spatially filtered to reduce noise at high spatial frequencies, and rotated to a reference angle. These dithered subimages were then co-added to produce a single image for each image number in Table 1 . Because the telescope operates on an altitude-azimuth mount, the characteristic diffraction spikes induced by the segmented primary and secondary spiders appear to rotate in equatorial coordinates during the night. In the mid-infrared, the relative intensity of the diffraction spikes is small, roughly $1 \%$ of the peak intensity of the PSF. In the case of NGC 1068, each image was rotated to equatorial north. For the PSF images, each raw image was either rotated to equatorial north, for purposes of creating the deconvolved images of NGC 1068 or, for purposes of creating the deconvolved images of the PSF, rotated to a common elevation angle thus keeping the orientation of the diffraction spikes fixed.

\subsection{Image Deconvolution}

The images were rebinned to a finer pixel scale $\left(0{ }^{\prime \prime} 034\right.$ pixel $^{-1}$ ) and deconvolved using a Richardson-Lucy maximum likelihood solution subject to a positivity constraint as developed by Richardson (1972) and Lucy (1974). The deconvolved images of NGC 1068 and the PSF are shown in Figure 1. As the deconvolved image depends critically on the stability of the PSF image, the matching of the PSF calibrator in elevation angle and the interleaved observations of NGC 1068 and the PSF should give optimal results.

To estimate the stability of the PSF image, one image was formed from the normalized sum of the first, third, and fifth images of the PSF from Table 1, and a second image was formed from the normalized sum of the second and fourth images from Table 1. This linear combination of PSF images results in a small effective difference in elevation angle between the two combined images. The deconvolution of the first of these combined PSF images by the second results in the compact images of the PSF shown in the third column of Figure 1 (FWHM 0"1). The combined image of HR 0911 obtained on October 3 was also deconvolved by the combined image of HR 0911 obtained on October 1, with an excellent result and negligible residual features. A more detailed discussion of various tests made to verify the deconvolution procedure is given in Appendix A.

MIRLIN provides subcritical spatial sampling of the PSF only for wavelengths $\lambda \leq 12 \mu \mathrm{m}$. Because the image deconvolution requires critically sampled images, all of the unprocessed images taken at 7.9 and $10.3 \mu \mathrm{m}$ were first smoothed by convolving them with a two-dimensional Gaussian function of FWHM 0"2 before proceeding with the data reduction. This smoothing has the effect of degrading the intrinsic spatial resolution at 7.9 and $10.3 \mu \mathrm{m}$ to the intrinsic spatial resolution at $12.5 \mu \mathrm{m}$.

\subsection{Photometry}

Photometric measurements were made of the raw images before any deconvolution algorithms were applied. Photometry was based on the measurements of HR 1457 and HR 0911, the magnitudes of which were determined by a combination of an intercomparison of several measurements taken on several nights, by an extrapolation based on their effective temperature, and by a consistency with the IRAS 12 and $25 \mu \mathrm{m}$ measurements. The magnitudes adopted at wavelengths $7.9,10.3,12.5$, and $24.5 \mu \mathrm{m}$ are $-2.99,-3.04$, -3.07 , and $-3.03 \mathrm{mag}$ for HR 1457 and $-1.86,-1.89$, -1.92 , and $-1.96 \mathrm{mag}$ for HR 0911. The "sky" was taken as an annulus 4".6-6".0 in diameter. The conversion from magnitude to flux density followed the prescription given in the Explanatory Supplement to the IRAS Catalog (Beichman et al. 1985); i.e., $0.0 \mathrm{mag}=63.2,38.0,26.2$, and $7.0 \mathrm{Jy}$ at $7.9,10.3,12.5$, and $24.5 \mu \mathrm{m}$.

\section{RESULTS}

\subsection{Imaging}

The combined images of NGC 1068 and of the PSF calibration stars are given as the "raw images" displayed in Figure 1. The PSF was diffraction limited at 12.5 and 24.5 $\mu \mathrm{m}$, varying from a FWHM of 0.3 at $12.5 \mu \mathrm{m}$ to 0.6 at 24.5 $\mu \mathrm{m}$, with the Airy diffraction rings apparent in each of the raw images. At 7.9 and $10.3 \mu \mathrm{m}$, the FWHM was $\sim 0.4$ due primarily to the smoothing described in $\S 3.2$. The ratio of the peak flux density per pixel to the noise level in the raw images varies from $\sim 300$ in the $24.5 \mu \mathrm{m}$ image to $\gtrsim 1000$ in the other three images. 
TABLE 2

PHOTOMETRY FOR NGC 1068

\begin{tabular}{cccc}
\hline \hline $\begin{array}{c}\text { Wavelength } \\
(\mu \mathrm{m})\end{array}$ & $\begin{array}{c}\text { Magnitude } \\
(\mathrm{mag})\end{array}$ & $\begin{array}{c}f_{v}^{\mathrm{a}, \mathrm{b}} \\
(\mathrm{Jy})\end{array}$ & $\begin{array}{c}\text { Magnitude Difference } \\
(\mathrm{mag})\end{array}$ \\
\hline $7.9 \ldots \ldots \ldots \ldots$ & 1.19 & $21 \pm 2$ & 0.04 \\
$10.3 \ldots \ldots \ldots$ & 0.46 & $25 \pm 2$ & 0.06 \\
$12.5 \ldots \ldots \ldots$. & -0.42 & $38 \pm 4$ & 0.08 \\
$24.5 \ldots \ldots \ldots$ & -2.58 & $75 \pm 8$ & 0.16 \\
\hline
\end{tabular}

a 4 " diameter beam.

${ }^{\mathrm{b}} f_{v}$ for 0.0 mag based on IRAS formulation (Beichman et al. 1985).

c Magnitude difference between photometry in a 4" and 2" diameter beam.

The four images were deconvolved as described above, and the four deconvolved images of NGC 1068 and of the PSFs, derived as described above, are given in Figure 1. The FWHM of the deconvolved PSF in the $12.5 \mu \mathrm{m}$ image is $00^{\prime \prime} 05$; that at $7.9,10.3$, and $24.5 \mu \mathrm{m}$ is $0.07,0$."10, and 0.12 . The uncertainties increase toward the center, to roughly $5 \%$ of the peak surface brightness at the peak in the case of the $12.5 \mu \mathrm{m}$ image. Based on the reproducibility of the deconvolved images described in Appendix A and Appendix B, the confidence in the reproducibility in the $12.5 \mu \mathrm{m}$ image extends to the minimum contour shown and is somewhat less at the other wavelengths. In the $7.9 \mu \mathrm{m}$ image the feature to the southwest is possibly spurious, as it does not reproduce in the first and second deconvolved images (see Fig. 8). A full accounting of the sources of uncertainty is given in Appendix A and Appendix B. Figure 8 demonstrates the reproducibility of the deconvolved images from independent data sets.

As seen in Table 1, the image taken at $12.5 \mu \mathrm{m}$ had the largest number of multiple redundant observations. It has the highest angular resolution and highest signal-to-noise ratio, i.e., dynamic range, of the four images obtained. For these reasons we have chosen to duplicate the deconvolved $12.5 \mu \mathrm{m}$ image in Figure $1 b$. We want to emphasize, however, that all four images, in particular that at $24.5 \mu \mathrm{m}$, show qualitatively the same features.

Each of the images is dominated by a central peak that contains one-third to one-half of the flux in a $4^{\prime \prime}$ diameter beam depending on the limits ascribed to the peak, which are ambiguous. The FWHM of the peak at $12.5 \mu \mathrm{m}$ is 0.26 ; the widths at the other wavelengths are comparable. The peak contains extended emission and, as shown below, is significantly more extended than can be accounted for by a pointlike source. The emission above $\sim 10 \%$ of the peak surface brightness is generally extended in a well-resolved north-south line (position angle measured east from north $\sim 10^{\circ}$ ) about $1^{\prime \prime}(70 \mathrm{pc})$ in length. Both the central peak and this extended emission are compact in the east-west direction. With the present deconvolution, they are formally slightly larger than the PSFs in the east-west direction; the measurements imply an intrinsic width of the peak at different wavelengths between $\sim 00^{\prime \prime} 05$ and $\sim 00^{\prime \prime} 1$. This is sufficiently small that we do not claim that we have resolved the peak in the east-west direction. At the different wavelengths, the peak blends smoothly into the clearly extended emission in the north-south direction. Based on a comparison of the raw images and reconvolved images, we estimate that for distances $\gtrsim 0$ ". 5 from the peak, the average uncertainty per pixel in the deconvolved images ranges from $\sim 4 \%$ of the peak value per pixel at $24.5 \mu \mathrm{m}$ to $\sim 1 \%$ in the other three images. The uncertainties generally increase toward the peak; see Appendix B for details.

The fainter extended emission follows a similar pattern in the four wavelengths bands. The northern extension appears to bend first slightly west, $\sim 0.35$ north of the infrared peak, and then, $\sim 00^{\prime \prime} 5$ north of the infrared peak, becomes more extended and bends east by $\sim 60^{\circ}$. The southern emission, which is less extended than the northern emission, also becomes more diffuse south of the largest infrared peak. A faint source appears $\sim 0$ ".75 to the northeast of the main infrared peak.

The deconvolved images in Figure 1 are qualitatively like the deconvolved images at $\sim 0$ ".2 deconvolved angular resolution from the Hale Telescope described by Bock et al. (1998). Both show a linear structure about $1^{\prime \prime}$ in the north-south direction that is unresolved in the east-west direction. The bend to the east present in the lower contours in Figure 1, however, is not apparent in the lower resolution images. Both the Keck and Palomar images are in general agreement with the deconvolved mid-infrared images from Braatz et al. (1993) with much lower angular resolution (deconvolved FWHM = 0"7 at $12.4 \mu \mathrm{m}$ ). Braatz et al. show an image that is oriented basically northeast by southwest, but their resolution is too low to distinguish shapes within the extension. The northeast orientation in their image may reflect a low-resolution observation of the bend seen at Keck. The images are also in qualitative agreement with the lower resolution images of Cameron et al. (1994), although the northeast elongation observed by these authors is resolved into at least two essentially linear features.

\subsection{Photometry}

The magnitudes of NGC 1068 within an artificial 4" diameter beam are given in Table 2. The uncertainties listed include both statistical and estimated calibration uncertainties. The flux densities reported here are similar to those reported in the literature. In view of the well-established dependence on beam size plus the known near-infrared variability in the luminosity of the nucleus of NGC 1068, which is as large as a factor of 2 in two decades at $3 \mu \mathrm{m}$ (see, e.g., Glass 1997), any small difference is not disturbing.

Photometric magnitudes of NGC 1068 were also obtained in a beam with a $2^{\prime \prime}$ diameter. The average differences between the $2^{\prime \prime}$ and $4 "$ diameter beams, which are not subject to many of the systematic uncertainties of the photometry, are also given in Table 2.

\section{DISCUSSION}

\subsection{Overview}

An overview of the energetics of NGC 1068 and how the present observations fit into the luminosity of NGC 1068 is provided by Figure 2, which compares the flux per octave of the Keck observations with the overall spectral energy distribution measured from NGC 1068. The latter has been determined from the published literature and reflects large beam measurements such as the IRAS measurements. Figure 2 establishes that the wavelengths used in the present observations sample the bulk of the emission from NGC 1068.

The arrows in Figure 2 indicate upper limits on the flux per octave measured in a Gaussian of FWHM equaling the east-west width of NGC 1068. In the four wavelength bands, they represent $35 \%, 14 \%, 21 \%$, and $21 \%$ of the total 


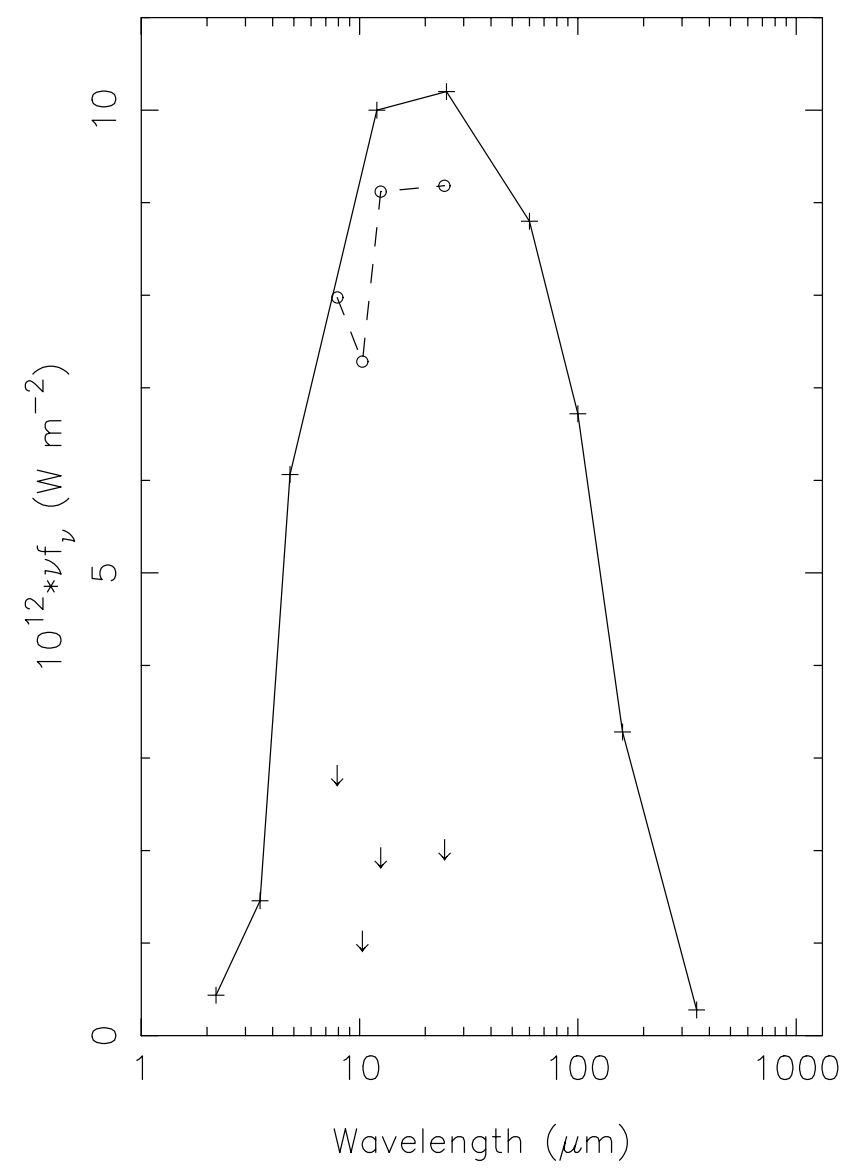

FIG. 2.-Spectral energy distribution of NGC 1068 within a 4" diameter beam resulting from the present observations (open circles; dashed line) is presented along with representative measurements from the published literature (Neugebauer et al. 1971; Marco \& Alloin 2000; Penston et al. 1974; Telesco et al. 1984; Thronson et al. 1987; Benford 1999; Joint IRAS Science Team 1989). The latter compilation is not meant to be exhaustive and represents various sizes of big beam measurements and includes the emission from the " $3 \mathrm{kpc}$ ring" (Telesco et al. 1984). The arrows represent conservative upper limits on the flux in a point source located at the core of NGC 1068 in the deconvolved images.

and represent estimates of the emission that can be ascribed to an unresolved point source. The estimate is an upper limit since the width of NGC 1068 is certainly greater than the FWHM of the deconvolved PSF. We are forced to the conclusion that only a small fraction of the observed luminosity in NGC 1068 comes from a pointlike nucleus; even in close to the central peak, there is a significant component of extended emission.

Emission from the low surface brightness disk $\sim 35^{\prime \prime}$ in diameter corresponding to a $3 \mathrm{kpc}$ diameter ring of molecular clouds in which star formation is occurring is included in the overall fluxes in Figure 2 (Telesco et al. 1984). Telesco et al. conclude that about half the bolometric luminosity of NGC 1068 comes from the $3 \mathrm{kpc}$ ring and estimate that at $10 \mu \mathrm{m} \sim 20 \%$ of the observed flux density comes from the disk. The disk is much colder than the nuclear source; Telesco et al. estimate that the $8-25 \mu \mathrm{m}$ radiation from the disk is only about $20 \%$ of the total. In contrast, at $100 \mu \mathrm{m}$, most of the emission is from the disk, and only a small fraction is from the nucleus. From the Keck data and Figure 2 we estimate that $\sim 2 / 3$ of the bolometric luminosity comes from the central area with 4 " diameter; i.e., the
$3 \mathrm{kpc}$ ring contributes only $\sim$ one-third of the bolometric luminosity. This apparent discrepancy between the Keck data and the data of Telesco et al. can be attributed to the uncertainties in the models and in the calibrations, including the wide bandwidths, plus the inherent difficulties of observations in the far-infrared. It is best resolved with high spatial resolution observations at 60 and $100 \mu$ m such as will be available with the SOFIA mission. Since it does not affect the following discussion, it will not be pursued further here.

\subsection{Comparison with [O III] and Radio Emission}

Figure 3 shows the $12.5 \mu \mathrm{m}$ contour map from the Keck observations superimposed on the HST [O III] image of Macchetto et al. (1994). Since no absolute astrometry was obtained for the Keck images, the juxtaposition of the infrared central peak with the apex is uncertain. The midinfrared peak was located at the scattering center as determined by Kishimoto (1999) who has recently carried out a reanalysis of the polarization data (Capetti, Macchetto, \& Lattanzi 1997) to determine the position of the nucleus of NGC 1068 as defined by ultraviolet polarimetry. Independent determinations of the position of the nucleus from astrometry at near-infrared wavelengths (Thatte et al. 1997) and mid-infrared wavelengths (Braatz et al. 1993) agree within the quoted uncertainties. With the choice of Kishimoto's central position, the line of the $12.5 \mu \mathrm{m}$ emission lies just inside the ionization cone, adjacent to one "wall." Also, this choice of alignment gives a good correlation between the mid-infrared emission and [O III] clouds $\mathrm{B}-\mathrm{F}$ and thus provides independent confidence in the efficacy of the adopted deconvolution.

The $12.5 \mu$ m contour is superimposed on the $5 \mathrm{GHz}$ map of Gallimore et al. (1996a) in Figure 4. Again, since no absolute astrometry was obtained for the Keck images, the juxtaposition of the infrared central peak with the radio features is uncertain. A shift of 0.3 would more completely overlay the mid-infrared features with the radio jet and cannot be ruled out. The $12.5 \mu \mathrm{m}$ central peak has been located on S1, the brighter of the southern components contained in the radio nucleus and the source Gallimore et al. identify with the center of NGC 1068. It has a flatter radio spectrum than the other radio sources and is the location of the torus observed by Gallimore et al. (1997). The qualitative agreement of the central portions is striking. Both images show a bend to the east, although the infrared bend is further to the north than the radio bend.

\subsection{Comparison with 3.5 and $4.8 \mu \mathrm{m}$ Observations}

At the brighter levels, the appearance of the present midinfrared images agrees qualitatively with the near-infrared images of Marco \& Alloin (2000) in showing extended emission along the north-south direction, the preferential direction of the axis of the radio emission and of the ionizing cone. As in the mid-infrared, the central core is unresolved in the near-infrared images (FWHM $\leq 00^{\prime \prime} 12$ ).

The agreement at the lower intensity contours is not so good. At both 3.5 and $4.8 \mu \mathrm{m}$, Marco \& Alloin (2000) see evidence both $\sim 0$ ". 5 east and west of the central peak for a putative torus at an intensity level roughly $1 \%$ of the peak surface brightness observed. The flux density at $4.8 \mu \mathrm{m}$ of the clumps defining this torus is $\sim 0.15 \mathrm{Jy}$. No sign of this torus is seen in the present mid-infrared observations. If the radiation is thermal, at a reasonable temperature, it should 


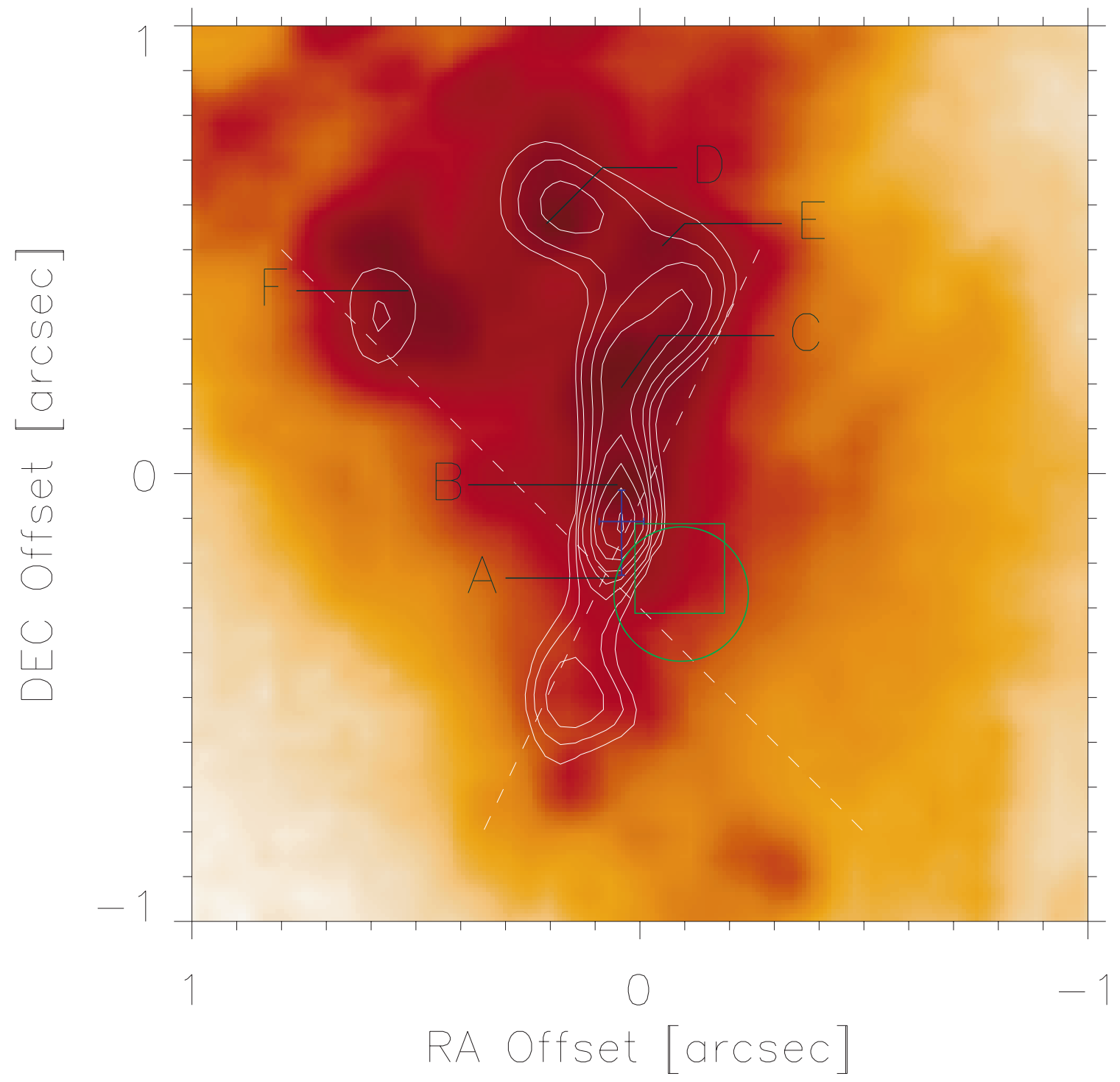

FIG. 3. - Contour plot of the $12.5 \mu \mathrm{m}$ deconvolved image is overlaid on the $\mathrm{O}$ [III] image of Machetto et al. (1994). The contours go from 0.9 of maximum in multiplicative steps of a factor of 2 as in Fig. $1 a$. No absolute astrometry was obtained for the Keck images; the juxtaposition of the infrared core with the [O III] image was obtained by aligning the mid-infrared peak with the position of the nucleus determined by ultraviolet polarimetry (Kishimoto 1999) shown by the blue cross. The letters label the local maxima in the [O III] emission as described by Evans et al. (1991). The somewhat subjective boundary of the conical region, denoted by dashed lines, is drawn to include [O III] clouds in the southern cone with strong polarization. Determinations of the position of the nucleus at near-infrared wavelengths (Thatte et al. 1997) and mid-infrared wavelengths (Braatz et al. 1993) are shown by the green square and circle, respectively. These independent determinations of the position of the nucleus agree within the quoted uncertainties, designated by the size of the symbols.

also be present in the $7.9 \mu \mathrm{m}$ deconvolved image. Tests made by placing an artificial point source 0.5 from the central peak of NGC 1068 indicate that at $7.9 \mu \mathrm{m}$ a source with a flux density of $\sim 0.1 \mathrm{Jy}$ or $0.5 \%$ of the total observed flux density at $7.9 \mu \mathrm{m}$ could easily be seen; see Appendix B. Calculations using the thermal parameters of Draine \& Lee (1984) show that ordinary astronomical graphite grains emitting $0.1 \mathrm{Jy}$ at $7.9 \mu \mathrm{m}$ would have to be hotter than 600 $\mathrm{K}$ to emit $0.15 \mathrm{Jy}$ at $4.8 \mu \mathrm{m}$. Ordinary astronomical graphite grains reradiating thermal radiation after being heated by a source with a luminosity of $1.5 \times 10^{11} L_{\odot}$ and $35 \mathrm{pc}$ distant would come to a temperature $\sim 245 \mathrm{~K}$. Thus, the grains cannot be heated purely by the central source unless the emissivities are vastly different from commonly accepted values, or the lumps derive their energy from an entirely different source.

\subsection{Silicate Absorption}

Kleinmann, Gillett, \& Wright (1976) obtained a spectrum of NGC 1068 from 8 to $13 \mu \mathrm{m}$ with $\sim 2 \%$ spectral resolution in a $5^{\prime \prime}$ diameter beam, which demonstrated that NGC 1068 has a pronounced absorption feature centered at $\sim 9.7 \mu \mathrm{m}$ attributed to silicates. More recent ISO-SWS spectra by Sturm et al. (2000) have delineated a broad absorption feature extending from $\sim 8.6$ to $\sim 10.0 \mu \mathrm{m}$ which is centered at $9.4 \mu \mathrm{m}$ rather than $9.7 \mu \mathrm{m}$, but the spectrum is probably contaminated because of the large ISO beam. The wavelength range of the $10.3 \mu \mathrm{m}$ band used in the present observations overlaps about half of this absorption and thus provides a measure of any strong silicate absorption morphology. Figure 5 gives the spectral energy distribution at the five locations shown. These loca- 


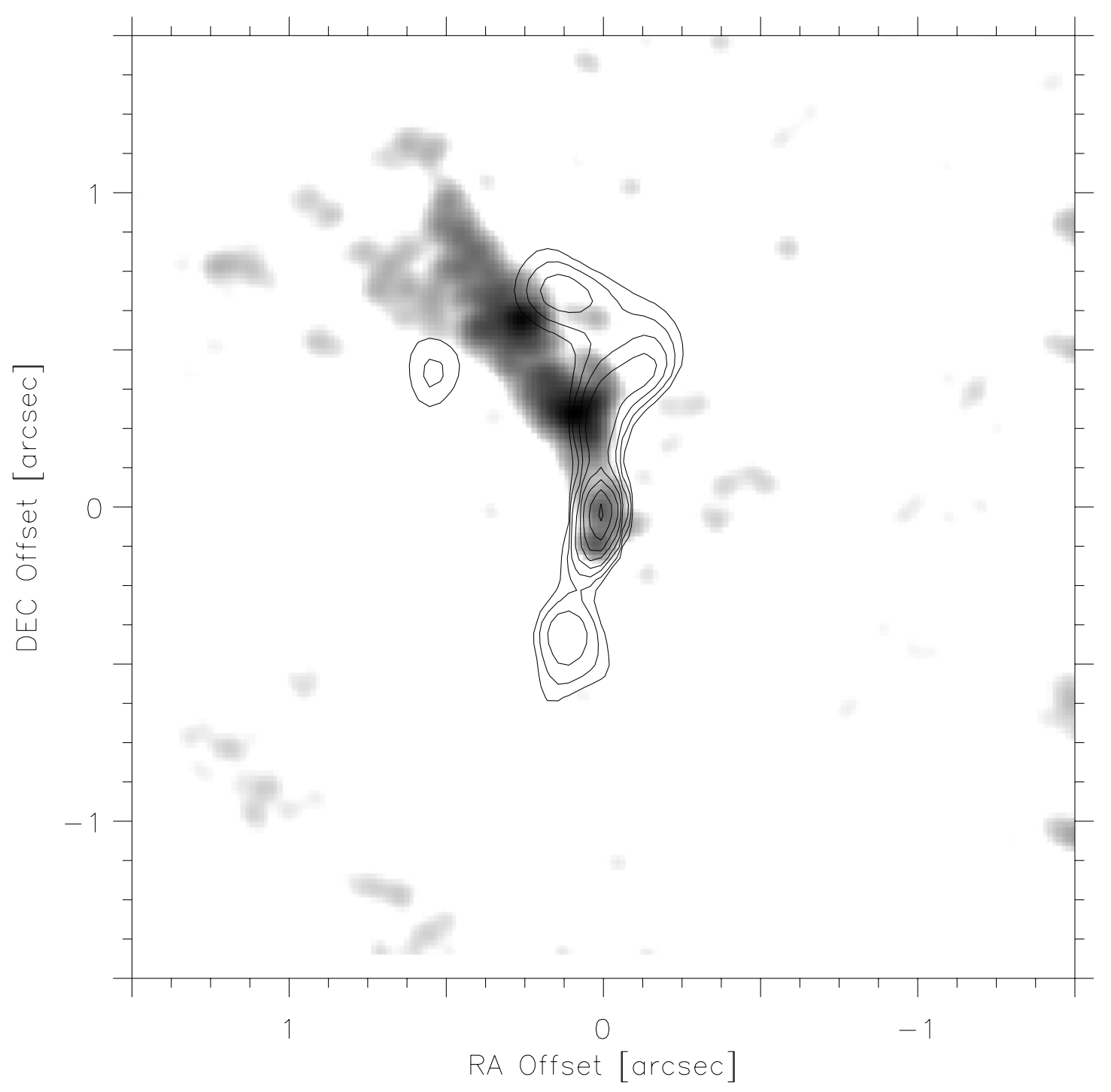

FIG. 4. - Contour plot of the $12.5 \mu \mathrm{m}$ deconvolved image is superimposed on the $5 \mathrm{GHz}$ map of Gallimore et al. (1996a). The contours are chosen as in Figs. $1 a$ and 3. The mid-infrared peak is aligned to the S1 radio source; no absolute astrometry was obtained for the Keck images. The position of source S1 coincides with the ultraviolet polarization center within the astrometric uncertainty (Kishimoto 1999; Capetti et al. 1997).

tions were chosen to approximate local maxima in the 12.5 $\mu \mathrm{m}$ image after the four deconvolved images of Figure 1 were smoothed to $\sim 00^{\prime \prime} 2 \mathrm{FWHM}$. It should be noted that, because the images at all four wavelengths were smoothed to the lowest resolution, this is a robust result.

The figure shows strong absorption on the peak and just to the south. There is a smooth continuum, perhaps even silicate emission, to the north and northeast. The most straightforward explanation is that we are seeing an attenuating screen of silicate that is more concentrated over the central peak of mid-infrared emission than in other regions. The absence of a silicate absorption feature in certain regions of the image could, of course, be due to purely geometrical effects such as the lack of temperature gradients along the line of sight.

\subsection{Temperatures}

In order to estimate the brightness temperature of the central peak, the source size was taken as that area that contained pixels in the deconvolved image with a flux density per pixel greater than half the maximum value. For the $12.5 \mu \mathrm{m}$ image, that with the best resolution, the size of this area was $\sim 0.4 \mathrm{pc}^{2}\left(0.006 \operatorname{arcsec}^{2}\right)$, and the resulting brightness temperature was $193 \mathrm{~K}$. Brightness temperatures for the other wavelengths, similarly defined, ranged from $118 \mathrm{~K}$ at $24.5 \mu \mathrm{m}$, with a source size $\sim 2.7 \mathrm{pc}^{2}$, to $205 \mathrm{~K}$ at $7.9 \mu \mathrm{m}$, with a source size $\sim 1.8 \mathrm{pc}^{2}$.

A $12.5-24.5 \mu \mathrm{m}$ color temperature was calculated from the deconvolved images at these wavelengths after first smoothing the deconvolved data to a common FWHM of 0 .'2. Only flux densities per pixel larger than 3 times the estimated noise per pixel, i.e., 0.03 times maximum flux density per pixel at $12.5 \mu \mathrm{m}$ and 0.12 times maximum flux density per pixel at $24.5 \mu \mathrm{m}$ were used. The resultant color temperatures, shown in Figure 6, are quite sensitive to the alignment of the two images, but in the central portion are $\sim 265 \mathrm{~K}$. The color temperature is highest in the central peak at $269 \mathrm{~K}$, and, significantly, there is no smooth dropping of the temperature along the extended north-south emission. In fact, the color temperature north of the central 

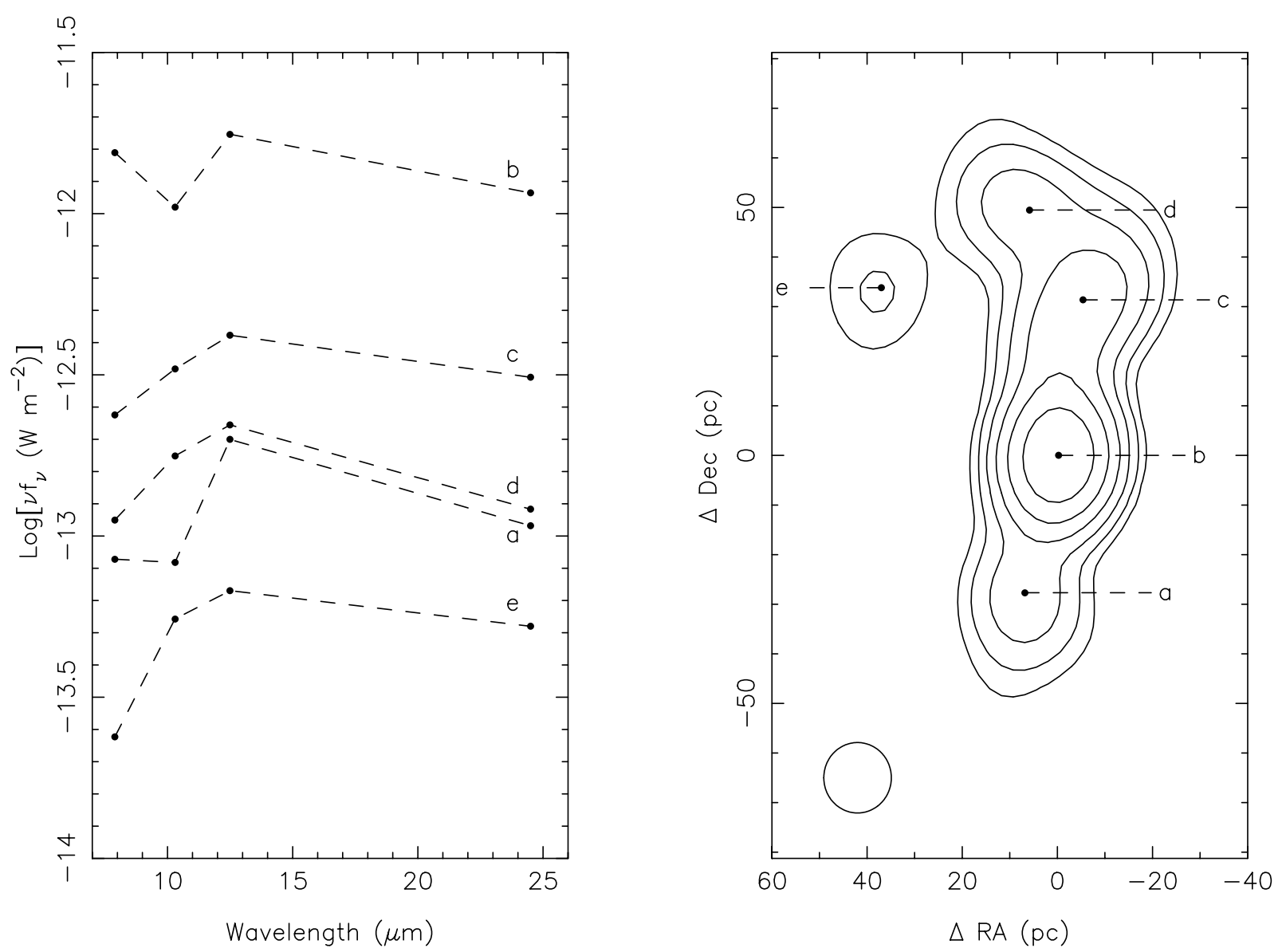

Fig. 5.- Spectral energy distribution in artificial beams of 0.2 diameter at five places in the $12.5 \mu \mathrm{m}$ deconvolved image is given in the left-hand panel. The locations of the photometry are shown in the right-hand panel superimposed on a contour plot of the $12.5 \mu \mathrm{m}$ deconvolved image smoothed to 0 ". 2 resolution. The contour levels are spaced by a multiplicative factor of 2 from the maximum; the size of the artificial beam is indicated. Each of the four deconvolved images of Fig. 1 were smoothed to similar resolution to make this comparison. Silicate absorption is identified with the $10.3 \mu \mathrm{m}$ data.

peak falls to $\sim 216 \mathrm{~K} \sim 0$ ".25 (20 pc) north of the peak and then rises again to $\sim 263 \mathrm{~K} \sim 0$ ". $4(30 \mathrm{pc})$ north of the peak.

Calculations using the averaged emissivity parameters of Draine \& Lee (1984) indicate that an optically thin cloud of pure silicate grains in thermal equilibrium with a central source of $1.5 \times 10^{11} L_{\odot}$ would have 12.5-24.5 $\mu \mathrm{m}$ color temperatures of 270 and $220 \mathrm{~K}$ at distances of 20 and $30 \mathrm{pc}$. If graphite grains were assumed, the color temperatures the same distances from the source would be 320 and $270 \mathrm{~K}$. The physical temperatures are predicted to be about 10-20 $\mathrm{K}$ cooler. Thus the measured color temperatures are in the range as expected for heating by a central source but do not show a smooth behavior with radial distance expected for a uniform distribution of dust grains in such a situation.

An obvious possibility is that the warm emission is from single-photon-heated small grains. This has, for example, been invoked in the starburst galaxy M82 to explain an increase in the color temperature with distance from the center (Telesco et al. 1991). Although this possibility cannot be excluded, we consider it unlikely since if it were the explanation for the warm emission, it would also be necessary to explain why, in Figure 5, there is no sign of the polycyclic aromatic hydrocarbon emission that is ubiquitous with single-photon heating.

Of course, beaming of a central source could increase the predicted temperature. An explanation for the results of
Antonucci \& Miller (1985) could invoke beaming of the central source. Baldwin, Wilson, \& Whittle (1987) have shown that the intensity of the central source could be as much as 200 times greater along the radio axis than to Earth. If $f$ is the "beaming factor" by which the luminosity of the central source in the direction of the dust cloud exceeds that toward Earth, the resultant temperature would be increased by a factor of $f^{1 / 4}$. Thus temperatures as high as $500 \mathrm{~K}$ could be obtained with beaming factors in the hundreds, although we do not consider such extreme beaming necessary.

\subsection{Interpretation}

The most prominent feature of the present observations is the highly asymmetric - almost linear - nature of the images, the well-resolved extent north-south, and the extreme narrowness east-west. A dominant feature of this emission is a relatively constant $12.5-24.5 \mu \mathrm{m}$ flux ratio or color temperature. We will designate the narrow lobe to the north of the dashed line in Figure $1 b$ as the "tongue." Also prominent is a "core," a name we assign to the region south of the dashed line in Figure $1 b$. We acknowledge that the dividing line between the two areas is arbitrary and is partially based on the model described below. The core contains about $2 / 3$ the flux per octave in the mid-infrared which we identify with the bolometric luminosity of the central 


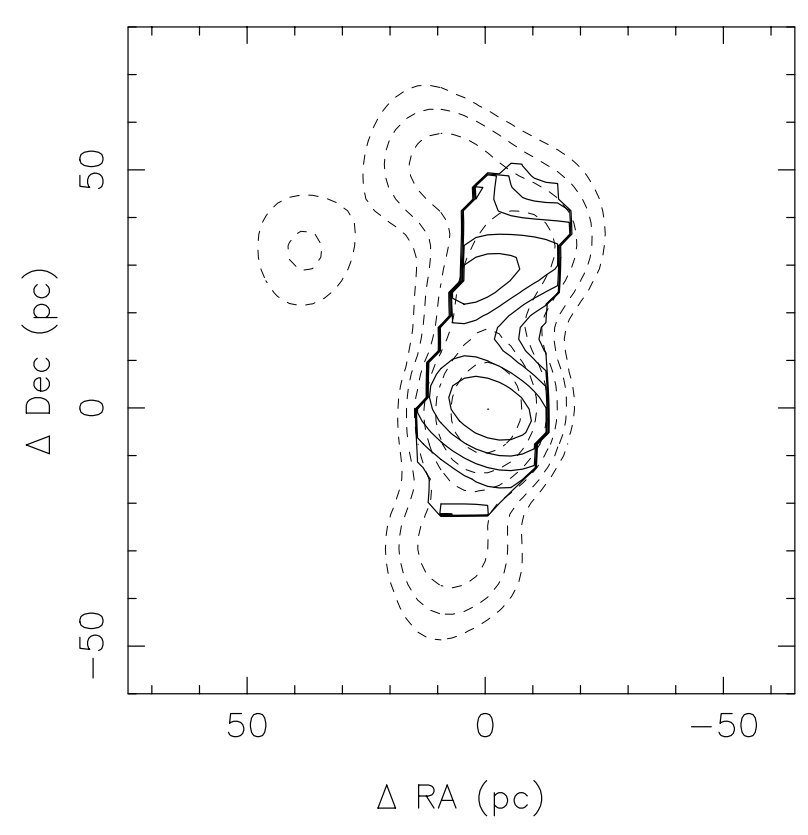

FIG. 6.-Color temperatures derived from the 12.5 and $24.5 \mu \mathrm{m}$ deconvolved images are shown. The deconvolved images were first smoothed to 0 ".2 to ensure comparable resolution in both images. The minimum flux density surface brightnesses used in obtaining the color temperatures were 0.03 times the maximum flux density pixel $^{-1}$ at $12.5 \mu \mathrm{m}$ and 0.12 times the maximum flux density pixel ${ }^{-1}$ at $24.5 \mu \mathrm{m}$. The contours go from a maximum of $269 \mathrm{~K}$ to a minimum of $169 \mathrm{~K}$ in intervals of $20 \mathrm{~K}$. The dashed contours represent the $12.5 \mu \mathrm{m}$ deconvolved image smoothed to 0 ". 2 resolution. The contour levels are spaced by a multiplicative factor of 2 from the maximum.

source within an area $4^{\prime \prime}$ in diameter, while the tongue contains about one-third. The core is aligned so its northpointing axis points in a direction slightly west of north while the emission in the tongue curves slightly east of north.

As a corollary to the feature noted above, we emphasize that the mid-infrared observations, by their small extent, convincingly associate a significant fraction, more than half, of the bolometric luminosity of NGC 1068 with an AGN rather than with starbursts. Our interpretation of the images is divided into a discussion of the core and a discussion of the tongue.

\subsubsection{The Core}

The core region is itself asymmetrical; the brightness of the emission in the north of the core is significantly greater than that to the south. The emission coming from the core can be understood if there is a very dense torus originating within a parsec or so of the AGN. Granato et al. (1997) have proposed a simple model consisting of a torus extending from $\sim 0.2$ pc to tens of parsecs. (Note, however, that this assumes that the torus has uniform density, whereas the HCN observations of, e.g., Tacconi et al. 1994 suggest that the gas observed at $\sim 100 \mathrm{pc}$ is quite inhomogeneous.) The asymmetric structure of the core image is a natural consequence of the inclination of the plane of the torus to the line of sight. We are seeing emission from the heated inside edge to the north, while the heated southern side is partly obscured by the torus itself. Granato et al. invoke an observation angle of $65^{\circ}$ and predict an asymmetry of $\sim 14: 1$ intensity ratio between the flux in a 0.2 diameter beam at the peak of the core and one in the lobe to the south. The observations yield a ratio of $\sim 10: 1$ for the flux density within a 0.2 diameter beam at the core and at the lobe to the south (positions $b$ and $a$ in Fig. 5). The predictions of Granato et al. (1997) give a separation of 0"3 between the core peak and the southern lobe, in good agreement with the measured separation of 0.4 .

Although the observations and models for the core region are in excellent agreement in some aspects, in others they differ. The models are clearly not attuned to the smallscale structure observed. The east-west width of the radiation pattern in the model of Granato et al. (1997) is significantly larger than observed. The modeled $10 \mu \mathrm{m}$ surface brightness has an east-west FWHM $\sim 0$ "15, while the deconvolved $12.5 \mu \mathrm{m}$ observations are barely, if at all, resolved east-west with an east-west FWHM $\lesssim 00^{\prime \prime} 05$. The modeled $10 \mu \mathrm{m}$ surface brightness drops to $10 \%$ at a full width of 0.4 , while in the deconvolved $12.5 \mu \mathrm{m}$ image the measured full width at $10 \%$ is closer to $0^{\prime \prime} 1$. The model clearly requires significantly deeper silicate absorption in the spectral energy distribution of the southern component compared to that of the northern source in the core. Figure 5 shows silicate absorption at both the southern locations (positions $a$ and $b$ ), but whether the extreme southern source displays the increased absorption required to attenuate the signal from the near side of the torus is uncertain, depending on the continuum temperature at the two locations. The high aspect ratio of the mid-infrared images, and whether the southern lobe we identify as part of the core is indeed associated with a torus, remain challenges for torus models to address.

\subsubsection{The Tongue}

The tongue is a powerful mid-infrared component in NGC 1068 associated with the western boundary of both the [O III] emission and the radio continuum emission (Evans et al. 1991; Macchetto et al. 1994; Gallimore et al. 1996a). On spectral grounds we identify this with reemission by dust of visual and ultraviolet radiation concentrated in an ionization cone which most likely originates in the nucleus of NGC 1068.

At this stage we note and comment upon three explanations for the formation of the ionization cone in NGC 1068.

1. Relativistic beaming.-The ultraviolet continuum could be produced nonthermally by a pair of relativistic jets and beamed along the jet direction through relativistic aberration. There is no evidence, however, that the jets associated with Seyfert galaxies, in contrast to those associated with spiral galaxies, are relativistic and we consider that this explanation is unlikely.

2. Dust absorption.-The ultraviolet continuum may be produced in a roughly isotropic source, such as an accretion disk, and cool dusty gas at low latitudes - the torus - then remove most of it, permitting escape only along the two polar directions. As the solid angle subtended by the ionization cone is $\sim 0.1$ of the sky, we would expect the power radiated by the torus to be at least 10 times that associated with the ionization cone. We have already shown, however, that the power reradiated at mid-infrared wavelengths from the northern ionization cone is one-third that from the core, and this is a lower bound on the luminosity flowing along the ionization cone. As the core luminosity is dominated by the mid-infrared power reported here, we conclude that the 
core is underluminous by at least an order of magnitude for dust absorption to account for the formation of the cones. (See Storchi-Bergmann, Mulchaey, \& Wilson 1992 for a related argument.)

3. Electron scattering.-Free electrons have a very high albedo at ultraviolet wavelengths, and a hot, ionized torus, probably orbiting inside a cool dusty torus will scatter most of the radiation from an isotropic ultraviolet source into the polar directions. This is our favored explanation for the ionization cone. (See Miller, Goodrich, \& Matthews 1991.) Note that the X-ray observations also suggest the presence of a hot torus.

The above picture is strengthened by the observations of Capetti et al. (1995) who measured wavelength-dependent, polarized visual, and near-ultraviolet continuum emission from the ionization cone, which appears to be concentrated at the edges of the radio emission (Capetti et al. 1997), just as in the mid-infrared observations reported here. This suggests that we are underestimating the luminosity of the ionization cone.

In view of the energetic considerations given above, it is appropriate to compare the potential luminosities with the Eddington luminosity of NGC 1068. The Eddington luminosity is based on the observations of Gallimore et al. (1996c) and Greenhill et al. (1996) who used water maser emission lines to estimate the mass of the black hole in NGC 1068. Unfortunately, the estimates of the two groups differ by a factor of 3 . Gallimore et al.'s measurements result in an Eddington luminosity of $1 \times 10^{12} L_{\odot}$, while those of Greenhill et al. give $3 \times 10^{11} L_{\odot}$. Both these luminosities are less than the putative isotropic bolometric luminosity of $\gtrsim 25 \times 10^{11} L_{\odot}$ derived above, but we can strongly discount this value only if we accept Greenhill et al.'s value of the black hole mass. It should be noted that either value of the Eddington luminosity is only somewhat larger than the total mid-infrared luminosity, suggesting that the accretion onto the black hole is almost Eddington-limited and that any gas within the ionization cone will be accelerated outward by radiation pressure as the flux there must exceed the Eddington flux.

The radiation pressure should also sweep the ionization cone clean of dust, although there is evidence (Miller et al. 1991) that the grains are continuously being replenished. The timescale for such clearing would be quite short, so an evacuated cone with dust at the edges might result. Thus, with the exception of the dust in a hypothesized central torus, the mid-infrared-emitting dust would be distributed in a thin shell around the surface of the ionization cone and would thereby appear as linear features since the line-ofsight path length is maximized at the edges of the shell. The observations would most likely imply a very patchy shell. This would obviate the need to invoke any beaming of the ultraviolet radiation that heats the dust, other than the relatively broad ionization cone.

Although the morphological resemblance of the radioand mid-infrared-emitting regions might suggest the radio jet being the heating source of the dust in the tongue, we argue that this is unlikely based on the energetics. Observation shows that the plasma responsible for the scattering of the AGN light is moving outward from the nucleus at a velocity of $300-1200 \mathrm{~km} \mathrm{~s}^{-1}$ (Miller et al. 1991). If we take this plasma velocity as the shock speed, mid-infrared emission can be produced by shocks in this flow through two dust-heating mechanisms: direct collisions between the plasma and dust particles (Draine 1981) and absorption of ultraviolet radiation produced by the postshock cooling plasma (cf. Dopita \& Sutherland 1996). However, unless the efficiency of converting the shock energy into infrared emission is extremely high, we cannot avoid the conclusion that this shock carries a mechanical luminosity comparable to the Eddington limit, which seems unlikely. There is no evidence that jets in Seyfert galaxies are this luminous. Furthermore, regardless of which mechanism dominates the dust heating, such a fast shock is bound to produce a substantial ultraviolet radiation. Therefore, the absence of strong ultraviolet sources near the mid-infrared-emitting region (recall that the ultraviolet radiation in the nuclear region is mostly scattered AGN light) also argues against the shock heating by the radio jet unless the ultraviolet radiation is perfectly shielded by dust.

Our picture, then, is of a thick, radiation-dominated accretion disk close to the central black hole that emits an ultraviolet power somewhat larger than the total midinfrared luminosity, which, primarily through electron scattering, with perhaps some dust absorption, is collimated into a pair of roughly antiparallel cones. It is likely that these cones are not just framed by a single accretion torus but are defined successively on many scales from the radius of the black hole to the radius of the narrow line region. Indeed, the partial occultation of the ionization cone by the dense, dusty gas that we are observing may be just a final stage in this process.

Why is the mid-infrared emission found mainly only on one side of the ionization cone and the radio jet? We suspect that this is because the gas flow on all scales may be quite irregular and the beam of the ionizing radiation is far from axisymmetric. Precession of the central accretion disk may also contribute to this outcome. This can also account for the otherwise surprising observation that the color temperature actually increases along the tongue. Suppose that the intensity in the northern ionization cone is quite nonuniform owing to the irregular scattering and absorption associated with its formation. The hotter dust observed near the end of the tongue might then be associated with an unusually intense pencil impacting a dense and dusty cloud, where the radiation flux is larger than the radiation flux that heats dust closer to the core despite the inverse square law dilution. In this way, we believe that we can account qualitatively for the observed morphology from radio to ultraviolet wavelengths.

If the interpretation presented above survives further scrutiny, it suggests an explanation as to why Seyfert galaxies (and most quasars) are radio-quiet (not silent). Specifically, the radiation drag close to the black hole and within the ionization cone may prevent the outflow from attaining ultrarelativistic speed. Future observations of other nearby Seyfert galaxies will be necessary to see if total luminosities are as large as found for NGC 1068.

In order to substantiate this picture, it would be useful to show that the AGN has sufficient X-ray, ultraviolet, and visual luminosity to provide for the observed mid-infrared luminosity. Observations of visual polarization, like those of Capetti et al. (1995), should be able to give this since it is presumably due to dust scattering. If we assume that the grains are similar to Galactic grains, their blue polarized flux should correlate with the mid-infrared flux. We can use the albedo and polarization expected from dust scattering 
to estimate the luminosity absorbed. This should give a lower limit on the luminosity in the ionization cone.

The luminosities measured in the present images are, as noted, close to the Eddington luminosity for NGC 1068. If the tight correlation between the bulge velocity dispersion and black hole mass (Ferrarese \& Merritt 2000; Gebhardt et al. 2000) is substantiated, black hole masses may be inferred in other galaxies from velocity dispersion measurements. By comparing the corresponding Eddington luminosity with the AGN luminosity derived from infrared observations similar to those presented here, we will be able to determine which AGNs are accreting at the Eddington rate. This should enhance our understanding of how black holes are fueled in galactic nuclei.

Finally, a consequence of the picture presented above is that a significant fraction of the luminosity of NGC 1068 is not associated with a torus per se but is produced by reprocessing radiation with dusty gas located $\sim 40 \mathrm{pc}$ away from the central source whose characteristics may be unique to NGC 1068. This complexity makes any interpretation of AGNs invoking a common morphology more difficult.

\section{SUMMARY AND CONCLUSIONS}

1. Mid-infrared observations of NGC 1068 at the diffraction limit of the Keck 10 meter Telescope show a bright core containing about one-third the total flux accompanied by north-south emission about $70 \mathrm{pc}$ long which is unresolved in the east-west direction. Thus most of the emission from the nuclear region of NGC 1068 comes from the elongated, highly asymmetric north-south structure.

2. At wavelengths between 7.9 and $24.5 \mu \mathrm{m}$, essentially all the nuclear flux of NGC 1068, as distinct from that in the $3 \mathrm{kpc}$ ring, is contained within a diameter of $300 \mathrm{pc}$ of the central core.

3. The $12.5-24.5 \mu \mathrm{m}$ color temperature of the radiation is $\sim 270 \mathrm{~K}$ and does not decrease smoothly with distance from the core but has a secondary maximum close to $270 \mathrm{~K}$ after dropping to $\sim 215 \mathrm{~K}$.

4. The absorption by silicates is lumpy even within the central region of NGC 1068. There is evidence for increased silicate absorption over the central core and to the south; there is less silicate extinction to the knots of emission to the north.

5. The low-level mid-infrared emission, when located using the scattering center determined by Kishmoto (1999), lies near one wall of the conical region depicted in [O III] (Evans et al. 1991; Macchetto et al. 1994), and features in the near-infrared emission correlate with clumps in the [O III] emission. The mid-infrared emission mimics the radio jet observed by Gallimore et al. (1996b).

6. No evidence for the putative outer torus present at 3.5 and $4.8 \mu \mathrm{m}$ (Marco \& Alloin 2000) is seen in these midinfrared images.
Two-thirds of the mid-infrared emission - the core-is interpreted as being the result of reradiation of the AGN luminosity by a dusty torus viewed at an angle so that we see one heated face almost directly while the other face is obscured. Most of the ultraviolet power of the nucleus appears to be redirected into the ionization cone by electron scattering. There are, however, significant details that need to be addressed before this picture can be accepted. The remaining one-third of the mid-infrared emission in NGC 1068 - the tongue - is associated with the western boundary of both the [O III] emission and the radio continuum emission. We identify this with reemission by dust of visual and ultraviolet radiation that originates in the core of NGC 1068 and is strongly beamed along the ionization cone.

The ability to perform ground-based imaging photometry of active galaxies in the mid-infrared is opening up several scientific opportunities. The most immediate is that observations such as these will greatly improve our estimates of the cosmological luminosity density of accreting black holes. X-ray observations have demonstrated that there is a large population of heavily obscured AGNs. X-ray observations, however, are not able to measure the absorbed power with any precision, and for this, infrared observations are crucial. We suspect that most reradiation by dust in obscured AGNs emerges in the mid-infrared. By contrast, most emission from star-forming regions may occur at longer wavelengths. In order to test this hypothesis, it will be necessary to perform additional, highresolution observations in the mid-infrared of active galaxies, such as those described here. If the hypothesis is confirmed, then SIRTF will be able to measure the reradiation in a much larger sample and provide the first accurate measurement of the luminosity density of active galaxies.

We thank the staff of the Keck Observatory, especially Bob Goodrich, for their assistance in making these observations possible. We also thank Pat Shopbell for discussions about the X-ray observations of NGC 1068 and Lee Armus, Bob Goodrich, Nick Scoville, Kris Sellgren, and Dave Thompson for discussions about various aspects of the interpretation. We thank Ski Antonnucci and Makoto Kisimoto for their helpful comments as referees. The W. M. Keck Observatory is operated as a scientific partnership between the California Institute of Technology, the University of California, and the National Aeronautics and Space Administration. It was made possible by the generous financial support of the W. M. Keck Foundation. B. T. S., G. N., K. M., and E. E. are supported by grants from the NSF and NASA. B. T. S. is supported by the SIRTF Science Center at Caltech. SIRTF is carried out at J.P.L., operated by Caltech under an agreement with NASA. This work was carried out in part (J. J. B., M. W. W., and M. E. R.) at J.P.L. The development of MIRLIN was supported by NASA's Office of Space Science.

\section{APPENDIX A}

\section{TESTS OF DECONVOLUTION}

There have been several, apparently conflicting, images of NGC 1068 in the infrared recently published. We therefore describe below in detail the tests performed on our images to characterize the statistical uncertainties and stability of the PSF and to uncover potential sources of systematic uncertainty. Because noise models are difficult to estimate in practice for 
deconvolved images, multiple redundant observations were used to make an assessment of the uncertainties. For example, from a comparison of the raw and reconvolved images, the differences between the images are found to be generally largest near the brightness peak, at a level significantly larger than the estimated statistical noise. This comparison implies that the uncertainties in the deconvolved images are dominated by variations in the shape of the PSF during the observations, as might be expected given the high signal-to-statistical noise ratio in the raw images.

Operationally, the uncertainty was characterized by comparing six deconvolved images based on six independent sets of NGC 1068 and PSF raw images (derived from the image pairs in Table 1). These deconvolved images (see Fig. 7) consistently reproduce the general features from the combined deconvolved image and the uncertainty over the image can be evaluated by comparison of these deconvolved images. As shown in Figure 7, the uncertainties are largest near the infrared peak and decrease with distance from the peak. The uncertainty in the combined image is $\sim 5 \%$ of the surface brightness.

As a check of the robustness of the deconvolved images, two images of NGC 1068 and PSF were constructed by combining data throughout the observations. The data were interleaved such that the effective air mass of the NGC 1068 and PSF were similar. The deconvolved images (NGC 1068 No. 1 and No. 2 in Fig. 8) are in excellent qualitative agreement with each other and the deconvolved images presented in Figure 1. The faint source to the northeast is present in all of the images at 10.3, 12.5, and $24.5 \mu \mathrm{m}$. The largest disagreement occurs at $7.9 \mu \mathrm{m}$, where the second image shows a rotated central elongation and a faint source, $1.4 \%$ of the peak, about $0^{\prime \prime} .2$ southwest of the nucleus. By so dividing, we see that the major features (east-west width, shape of the northern and southern extensions, relative strength of the peak brightness) are seen to be reproducible when the data are divided into individual images. Faint sources, such as the northeast source, generally appear in each of the deconvolved images in Figures 7 and 8. One exception is the faint source southwest of the nucleus at $7.9 \mu \mathrm{m}$, which is possibly spurious.

As a test of systematic uncertainty, PSFs were constructed from HR 1457 and HR 0911. The PSFs were mismatched both in average air mass $(\sim 1.15$ for HR 0911 and 1.03 for HR 1457) and in flux (HR 1457 is 2.8 times brighter than HR 0911). At $12.5 \mu \mathrm{m}$ it is also possible to deconvolve the October 1 data using the October 3 PSF observations. The deconvolution of the one PSF by the other is shown in Figure 8. We chose to deconvolve the PSF with larger average air mass by the PSF with the lower average air mass to illustrate the maximum error. Since the FWHM of the PSF observed at a lower air mass is always slightly smaller than that of the PSF with a higher air mass, the reverse deconvolution gives significantly better results. The deconvolved PSFs are ellipsoidal, with FWHM in the extended direction that ranges from 1.5 to 2 times that of the deconvolved PSFs presented in Figure 1. The largest spurious features in Figure 8 appear at 3\% of the peak brightness, owing to incomplete cancellation of the first Airy diffraction ring.

As a further test, images of NGC 1068 were deconvolved with a mismatched calibrator. As shown in Figure 8, a combined image of NGC 1068 observed in sequence with HR 1457, and deconvolved using the PSF from HR 0911 (NGC 1068 No. 3) was formed. The remaining observations of NGC 1068 were deconvolved using the PSF from HR 1457 (NGC 1068 No. 4). The two deconvolved images gave similar results, although the NGC 1068 image and the PSF were mismatched in air mass as much as possible and well separated in time. Finally, as a severe test of the systematic uncertainty, the image of NGC 1068 from the night of October 3 was deconvolved by the PSF from October 1 (NGC 1068 No. 5), and the October 1 observations of NGC 1068 were deconvolved by the PSF from October 3 (NGC 1068 No. 6). Although these results are less satisfactory, as
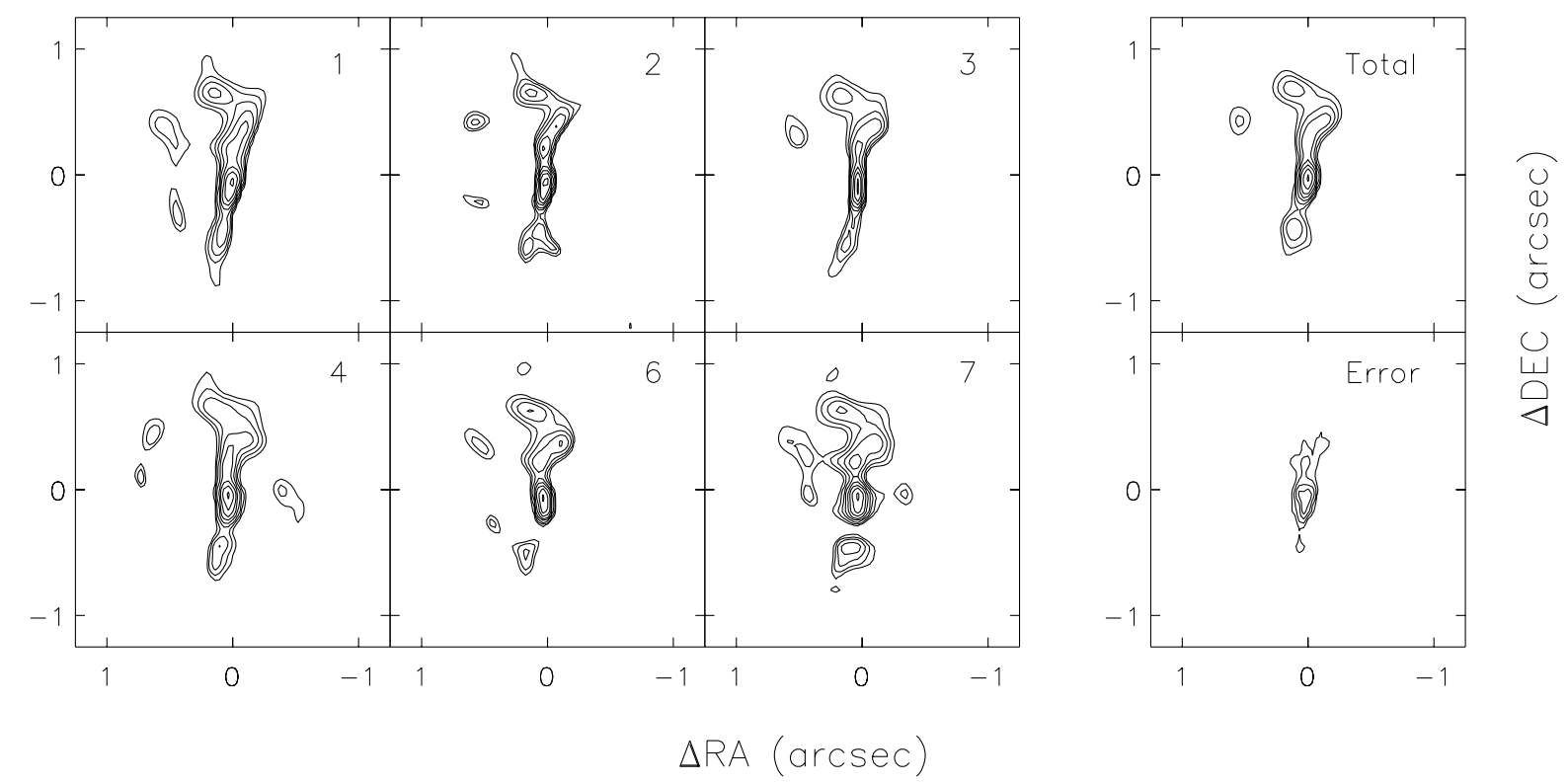

FIG. 7.-Deconvolved images based on pairs of raw NGC 1068 and PSF images at $12.5 \mu \mathrm{m}$ are shown. The labels correspond to the numbers of the image pairs in Table 1, with the bright star used as a PSF and the air masses are identified therein. Image 7 has the poorest matching between the air mass of NGC 1068 and that of the PSF. Contours are spaced by multiplicative factors of 2 as in Fig. 1. The combined image and estimated uncertainties are shown at the right with the same minimum contour. 
N 1068 Total N N N N 1068 \#1 \#2
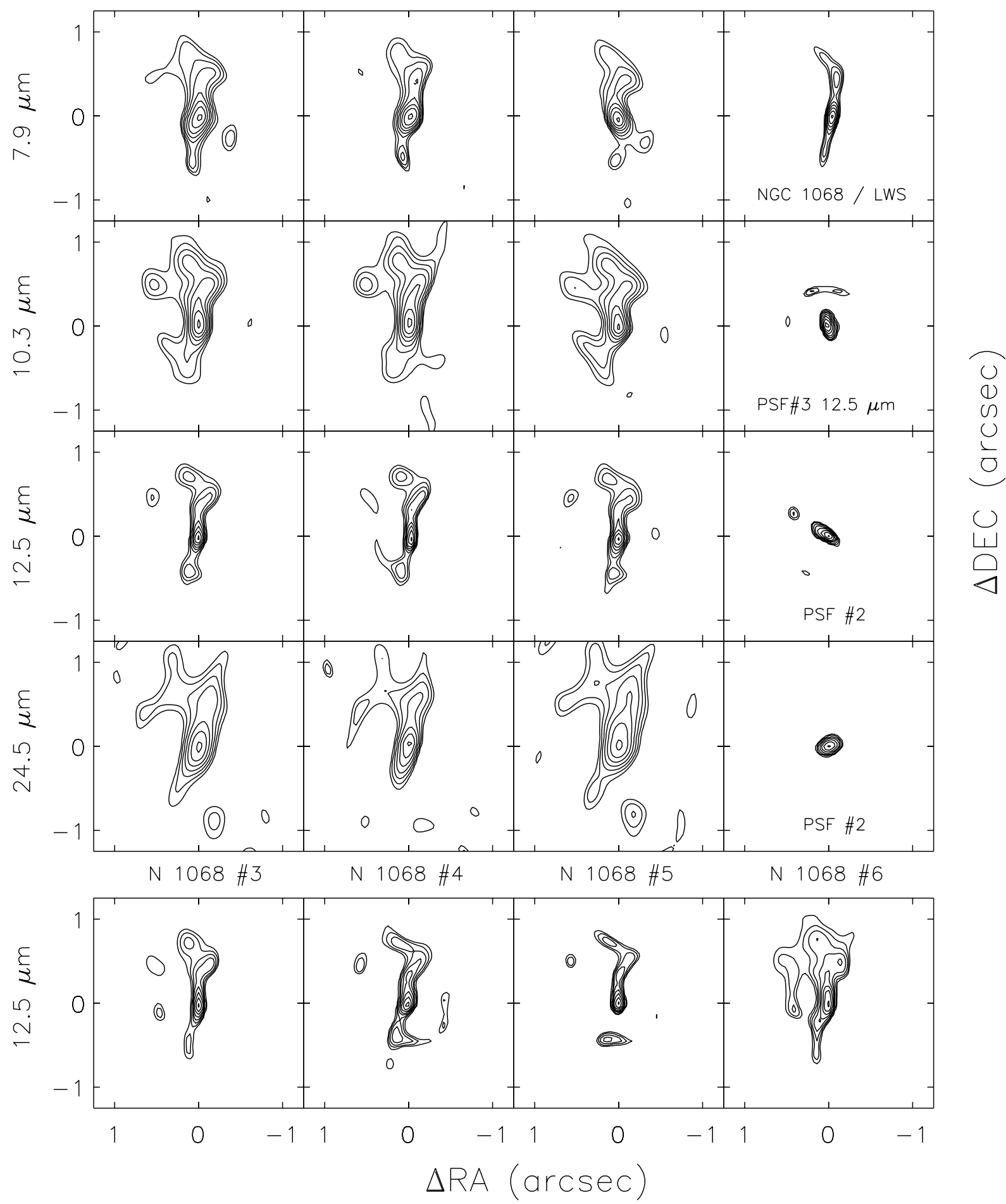

Fig. 8. - Comparison of deconvolved images derived from subsets of the observation log listed in Table 1 is given. The columns in the upper left-hand $3 \times 4$ panels are (NGC 1068 Total) the deconvolved image of NGC 1068 using the entire data set, (NGC 1068 No. 1 and NGC 1068 No. 2) deconvolved images of NGC 1068 using half of the NGC 1068 and PSF data. The data were interleaved to have similar average air masses for the NGC 1068 and the PSF observations. In the upper right-hand $1 \times 4$ panel, from top to bottom, are the deconvolved image of NGC 1068 obtained with the LWS instrument at a wavelength $\lambda \approx 7.9 \mu \mathrm{m}$ under nonideal seeing conditions, (PSF No. 3) the deconvolved PSF at $\lambda=12.5 \mu \mathrm{m}$ from the October 1 PSF deconvolved by the October 3 PSF, and (PSF No. 2) the deconvolved PSF from the HR 0911 PSF deconvolved by the HR 1457 PSF at $\lambda=12.5$ and $24.5 \mu \mathrm{m}$. In the bottom $4 \times 1$ panel: (NGC 1068 No. 3) the image of NGC 1068 obtained when HR 0911 was substituted as a PSF instead of HR 1457 using a combined image of NGC 1068 when HR 1457 was the PSF pair in Table 1, (NGC 1068 No. 4) the image of NGC 1068 obtained when HR 1457 was substituted as a PSF instead of HR 0911 using a combined image of NGC 1068 when HR 0911 was the PSF pair in Table 1, (NGC 1068 No. 5) the NGC 1068 deconvolution combining the NGC 1068 observations of October 3 and PSF observations from October 1, (NGC 1068 No. 6) the NGC 1068 deconvolution combining the NGC 1068 observation from October 1 with the PSF observation from October 3. 
might be expected, the general morphology is clearly preserved. Both these deconvolved images of the NGC 1068 and the PSF illustrate the maximum effect of systematic error due to changes in the PSF with time and elevation angle. Both the differential average air mass and time separation between raw image and PSF are many times better for the images presented in Figure 1.

As a final test, images of NGC 1068 and a PSF star (IRC $+00^{\circ} 032$ ) were obtained on 2000 January 27 using a different mid-infrared camera, the LWS (Jones \& Puetter 1993) on the Keck 110 meter Telescope. LWS provides Nyquist-sampling of the Airy function with 0 ."08 pixels. Unfortunately, on the night of the observations the seeing was approximately twice the diffraction-limited resolution. One hundred ninety-two observations, in six groups of 32 observations and each of 1 s exposure time, were taken of NGC 1068 interleaved with similar observations of the PSF star. The $10 \%$ of each set of $\sim 200$ observations with the smallest FWHM were selected and the images co-added; these co-added images were used to obtain a deconvolved image of NGC 1068 (Fig. 8) that bears a good resemblance to the images obtained with MIRLIN.

\section{APPENDIX B}

\section{TESTS OF SENSITIVITY TO ADDITIONAL SOURCES}

The detectability and stability of a source near the main peak in the deconvolved image may be tested by creating a synthesized image, whereby a faint point source is added to the raw NGC 1068 image. If the PSF is systematically different in the PSF and NGC 1068 images, the deconvolved synthesized image may be expected to change the flux density and position of the faint point source. A point source based on the deconvolving PSF with a flux density ranging from $10 \%$ to $0.5 \%$ of the flux density of NGC 1068 was placed at positions $\sim 0.5$ east and west of the nucleus. The point source was generally recovered in the deconvolved image with relatively little change in flux density or position down to the $0.5 \%$ level at $7.9,10.3$, and $12.5 \mu \mathrm{m}$ and down to the $2.0 \%$ level at $24.5 \mu \mathrm{m}$. These levels are consistent with the contours chosen in Figure 1 .

If the point source is inserted closer to the nucleus ( 0.3 to the east or west), the flux density and position are still recovered in the deconvolved image. The deconvolved resolution appears to degrade slightly so that peak surface brightness is about 2 times lower. These tests were also performed using an independent PSF (HR 0334) in the synthesized image. In this case, the resolution of the point source was somewhat lower, as expected. However, the flux density and position are recovered with similar accuracy as before. Based on these tests, the estimated uncertainty in the photometry of a source placed 0"3-0"6 east or west of the nucleus, evaluated in a $0.3 \times 0.3$ artificial beam, is greater than $0.5 \%$ of the total flux density at 7.9 and $12.5 \mu \mathrm{m}$.

Similar tests were also performed on the LWS images described in Appendix A with worse image recovery than with the MIRLIN data. The seeing conditions when the LWS data were taken were significantly poorer than when the MIRLIN data were obtained and limited the reliability of the LWS data.

Antonucci, R. R. J., \& Miller, J. S. 1985, ApJ, 297, 621

Baldwin, J. A., Wilson, A. S., \& Whittle, M. 1987, ApJ, 319, 84

Barthel, P. D. 1989, ApJ, 336, 606

Beichman, C. A., Neugebauer, G., Habing, H. J., Clegg, P. E., \& Chester, T. J. 1985, Infrared Astronomical Satellite (IRAS) Catalog and Atlases, Explanatory Supplement (Washington, DC:US Government Printing Office)

Benford, D. J. 1999, Ph.D. thesis, California Inst. of Tech.

Bock, J. J., Marsh, K. A., Ressler, M. E., \& Werner, M. W. 1998, ApJ, 504, L5

Braatz, J. A., Wilson, A. S., Gezari, D. Y., Varosi, F., \& Beichman, C. A. 1993, ApJ, 409, L5

Cameron, M., Storey, J. W. V., Rotactuc, V., Genzel, R., Verstraete, L., Drapatz, S., Siebenmorgen, R., \& Lee, T. J. 1993, ApJ, 419, 136

Capetti, A., Axon, D. J., Macchetto, F., Sparks, W. B., \& Boksenberg, A. 1995, ApJ, 446, 155

Capetti, A., Macchetto, F. D., \& Lattanzi, M. G. 1997, ApJ, 476, L67

Crenshaw, D. M., \& Kraemer, S. B. 2000a, ApJ, 532, L101

2000b, ApJ, 532, 247

Dopita, M. A., \& Sutherland, R. S. 1996, ApJS, 102, 161

Draine, B. T. 1981, ApJ, 245, 880

Draine, B. T., \& Lee, H. M. 1984, ApJ, 285, 89

Efstathiou, A., Hough, J. H., \& Young, S. 1995, MNRAS, 277, 1134

Evans, I. N., Ford, H. C., Kinney, A. L., Antonucci, R. R. J., Armus, L., \& Caganoff, S. 1991, ApJ, 369, L27

Ferrarese, L., \& Merritt, D. 2000, ApJL, in press (astro-ph/0006053)

Gallimore, J. A., Baum, S. A., \& O'Dea, C. P. 1996a, ApJ, 464, 198

. 1997, Nature, 388, 852

Gallimore, J. F., Baum, S. A., O’Dea, C. P., \& Pedlar, A. 1996b, ApJ, 458, 136

.1996b, ApJ, 462, 740

Gebhardt, K., et al. 2000, ApJL, in press (astro-ph/0006289)

Glass, I. S. 1997, Ap\&SS, 248, 191

Granato, G. L., Danese, L., \& Franceschini, A. 1997, ApJ, 486, 147

Greenhill, L. J., Gwinn, C. R., Antonucci, R., \& Barvainis, R. 1996, ApJ, 472, L21

\section{REFERENCES}

Iwasawa, K., Fabian, A. C., \& Matt, G. 1997, MNRAS, 289, 443

Joint IRAS Science Team. 1989, IRAS Point Source Catalog, Version 2 (Washington, DC: US Government Printing Office)

Jones, B. \& Puetter, R. C. 1993, Proc. SPIE, 1946, 610

Kishimoto, M. 1999, ApJ, 518, 676

Kleinmann, D. E., Gillett, F. C., \& Wright, E. L. 1976, ApJ, 208, 42

Koyama, K., Inoue, H., Tanaka, Y., Awaki, H., Takano, S., Ohashi, T., \& Matsuoka, M. 1989, PASJ, 41, 731

Kraemer, S. B., \& Crenshaw, D. M. 2000, ApJ, 532, 256

Krolik, J. H. 1999, Active Galactic Nuclei: From the Central Black Hole to the Galactic Environment (Princeton: Princeton Univ. Press)

Laurent, O., Mirabel, I. F., Charmandaris, V., Le Floc'h, E., Lutz, D., \& Genzel, R. 2000, ISO beyond the Peaks, in press (astro-ph/0003288)

Lucy, L. B. 1974, AJ, 79, 745

Macchetto, F., Capetti, A., Sparks, W. B., Axon, D. J., \& Boksenberg, A. 1994, ApJ, 435, L15

Marco, O., \& Alloin, D. 2000, A\&A, 353, 465

Matt, G., et al. 1997, A\&A, 325, L13

Matt, G., Fabian, A. C., Guainazzi, M., Iwasawa, K., Bassani, L., \& Malaguti, G. 2000, MNRAS, in press (astro-ph/0005219)

Miller, J. S., Goodrich, R. W., \& Mathews, W. G. 1991, ApJ, 378, 47

Neugebauer, G., Garmire, G., Rieke, G. H., \& Low, F. J. 1971, ApJ, 166, L45

Papadopoulos, P. P., Seaquist, E. R., \& Scoville, N. Z. 1997, ApJ, 477, 173

Penston, M. V., Penston, M. J., Selms, R. A., Becklin, E. E., \& Neugebauer, G. 1974, MNRAS, 169, 357

Pier, E. A., \& Krolik, J. H. 1993, ApJ, 418, 673

Planesas, P., Scoville, N., \& Myers, S. T. 1991, ApJ, 369, 364

Ressler, M. E., Werner, M. W., van Cleve, J., \& Chou, H. A. 1994, Exp. Astron., 3, 277

Richardson, W. H. 1972, J. Opt. Soc. Am., 62, 55

Rouan, D., Rigaut, E., Alloin, D., Doyon, R., Lai, O., Crampton, D., Gendron, E., \& Arsenault, R. 1998, A\&A, 339, 687

Schinnerer, E., Eckart, A., Tacconi, L. J., Genzel, R., \& Downes, D. 2000, ApJ, 533, 850

Storchi-Bergmann, T., Mulchaey, J. S., \& Wilson, A. S. 1992, ApJ, 395, L73 
Sturm, E., Lutz, D., Tran, D., Feuchtgruber, H., Genzel, R., Kunze, D., Moorwood, A. F. M., \& Thornley, M. D. 2000, A\&A, in press (astro$\mathrm{ph} / 0002195)$

Tacconi, L. J., Genzel, R., Blietz, M., Cameron, M., Harris, A. I., \& Madden, S. 1994, ApJ, 426, 77

Telesco, C. M., Becklin, E. E., Wynn-Williams, G. C., \& Harper, D. A. 1984, ApJ, 282, 427

Telesco, C. M., Joy, M., Dietz, K., Decher, R., \& Campins, H. 1991, ApJ, 369,135
Thatte, N., Quirrenbach, A., Genzel, R., Maiolino, R., \& Tecza, M. 1997, ApJ, 490, 238

Thronson, H. A., Walker, C. K., Walker, C. E., \& Maloney, P. 1987, ApJ, 318,645

Tully, R. B. 1988, Nearby Galaxies Catalog (New York: Cambridge Univ. Press)

Ueno, S., Mushotzky, R. F., Koyama, K., Iwasawa, K., Awaki, H., \& Hayashi, I. 1994, PASJ, 46, L71

Weinberger, A. J., Neugebauer, G., \& Matthews, K. 1999, AJ, 117, 2748 